\title{
Spatiotemporal Control to Eliminate Cardiac Alternans Using Isostable Reduction
}

\author{
Dan Wilson and Jeff Moehlis \\ Department of Mechanical Engineering, University of California, Santa Barbara, CA 93106
}

\begin{abstract}
Cardiac alternans, an arrhythmia characterized by a beat-to-beat alternation of cardiac action potential durations, is widely believed to facilitate the transition from normal cardiac function to ventricular fibrillation and sudden cardiac death. Alternans arises due to an instability of a healthy period-1 rhythm, and most dynamical control strategies either require extensive knowledge of the cardiac system, making experimental validation difficult, or are model independent and sacrifice important information about the specific system under study. Isostable reduction provides an alternative approach, in which the response of a system to external perturbations can be used to reduce the complexity of a cardiac system, making it easier to work with from an analytical perspective while retaining many of its important features. Here, we use isostable reduction strategies to reduce the complexity of partial differential equation models of cardiac systems in order to develop energy optimal strategies for the elimination of alternans. Resulting control strategies require significantly less energy to terminate alternans than comparable strategies and do not require continuous state feedback.
\end{abstract}

\section{INTRODUCTION}

The elimination of cardiac arrhythmia is a fundamental challenge in the field of cardiology. The most lethal of these arrhythmias, known as ventricular fibrillation, represents an interruption of the spatially coordinated cardiac dynamics responsible for pumping blood throughout the body. Ventricular fibrillation, commonly referred to as cardiac arrest, results from pathological, self-sustaining spiral waves [61], [3], [21]. Once cardiac fibrillation begins, the application of a high intensity shock is the only clinically reliable way of quickly restoring normal function [49], [54], [35], and failure to do so within minutes can result in death.

The transition from normal cardiac functioning to ventricular fibrillation is often facilitated by the existence of cardiac alternans, the beat-to-beat alternation of dynamic cardiac behavior at a constant rate of depolarization [51]. At a cellular level, alternans usually manifests in alternating long and short action potential durations (APDs), defined as the length of time the transmembrane voltage remains above resting potential after the cell fires (see e.g. the bottom panel of Figure 1). While alternans can be present during healthy cardiac conditions, they have long been linked with the onset of sudden cardiac death [51]. It is widely believed that the development of alternans can facilitate the dispersion of refractoriness in cardiac tissue known as discordant alternans, [44], [55], [56], [4], [11] producing favorable conditions for the formation of reentrant spiral waves.

In the first part of this work, we consider alternans on a one-dimensional ring of cardiac tissue

$$
C_{m} \frac{\partial V(r, t)}{\partial t}=D \frac{\partial^{2}}{\partial r^{2}} V(r, t)+\left(-I_{\text {ion }}(r)+I_{\text {stim }}(r, t)\right),
$$

where $V$ represents the transmembrane potential, $D=1.5 \mathrm{~cm}^{2} / \mathrm{s}$ is the diffusion constant, $I_{\text {ion }}(r)$ gives the membrane current density, $I_{\text {stim }}(r, t)$ is an external current controlled input, $C_{m}=1 \mu F / \mathrm{cm}^{2}$ is the membrane capacitance, and $r$ gives the position around the ring. While the diffusive term in (1) is linear, the underlying ionic currents are, in general, nonlinear functions of the underlying cellular dynamics. In this study, we consider the Fox model of canine cardiac tissue [16] for membrane current density and other gating variables. We take all parameters to be nominal. Equation (1) has periodic boundary conditions, $V(r+l)=V(r)$, and for a long enough ring the tissue admits traveling wave solutions. If we let the length of the ring be $l=10 \mathrm{~cm}$, and create a traveling wave moving in the positive $r$ direction in the medium, we find alternans develop in the system, as shown in Figure 1. Note that on short time scales shown in the top-right panel, the dynamics look as though they display period-2 alternant behavior. On longer time scales, as shown in the bottom panel, the severity of the alternant behavior at any given location waxes and wanes due to the complicated interplay between action potential and conduction velocity restitution; the resulting quasiperiodic dynamics have been observed in experimental preparations [17] and have been investigated in detail from a theoretical perspective [25], [8], [9].

(C) 2016. This manuscript version is made available under the Elsevier user license

http://www.elsevier.com/open-access/userlicense/1.0/ 

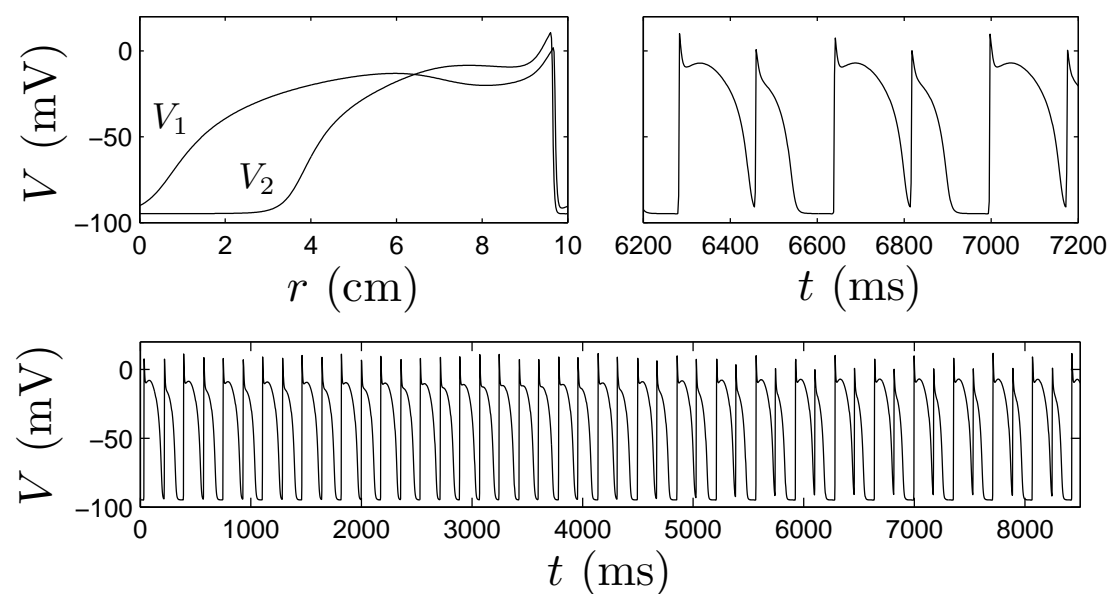

FIG. 1. The top-left panel shows spatially concordant alternans throughout the ring. The snapshots $V_{1}$ and $V_{2}$ are $175 \mathrm{~ms}$ apart, approximately the time it takes for the wave to travel once throughout the medium. In the snapshot $V_{1}$, the action potentials last much longer, while in the snapshot $V_{2}$, the cells repolarize quickly. The top-right panel shows the voltage profile of a single cell in the medium at $r=5 \mathrm{~cm}$, showing that alternans are also present at the cellular level. The bottom panel shows the voltage profile of the same cell on a longer time scale to illustrate the complicated quasiperiodic dynamics of the instability in the ring which is characterized in [25], [8], [9].

Because alternans may promote the formation of spiral wave reentry, termination of this arrhythmia could be useful from a clinical standpoint as a method of prevention for those who are susceptible to cardiac fibrillation. However, the underlying dynamics associated with the membrane current density make (1) difficult to work with directly to devise alternans elimination strategies. Indeed, experimentally successful alternans elimination strategies have utilized model independent state feedback methodologies which do not explicitly take into account the cellular dynamics but rather drive the system to an underlying unstable period-1 orbit [46], [23], [5], [27]. While such strategies are useful when the system dynamics are difficult to work with or have parameters which are not fully known, they ignore important features, likely leading to a sacrifice in energy efficiency of the control strategy. Other control strategies employ state feedback techniques which may be difficult to implement in real time in an in vivo setting [19], [18]. As a middle ground between these two strategies, in this work we will approach this control problem from the perspective of isostables [36], [59] which preserves important information about (1) but is still simple enough to be treated analytically.

For excitable systems such as (1) the notion of isostables, defined to be sets of initial conditions that share the same asymptotic convergence towards a fixed point [36], provide a convenient means of understanding the underlying system dynamics (for a brief introduction on the concept of isostables in a two-dimensional excitable ODE, we refer the reader to Appendix A. For a more complete discussion about isostables, we refer the reader to [36] and [60]). Isostables of excitable systems are analogous to isochrons of systems with stable periodic orbits [61], [33], [24], [13], as both have the ability to work with a complicated and high dimensional nonlinear system in terms of a reduced system with a single variable. When working with isostables, the resulting isostable reduction [59], [60] captures the effect of perturbations to the state variables on the isostable coordinates. Isostable reduction is similar to reduction methods which use inertial manifold [7], [15], or center manifold [48] theory. However, a significant advantage of isostable reduction is that the dynamics are not required to rapidly collapse onto a lower dimensional manifold (see e.g. [60]).

At a cellular level, alternans is caused by the existence of a stable period-2 orbit; in [59] we found that it was possible to use a control algorithm based on an isostable reduction to eliminate alternans by stabilizing the underlying unstable period-1 orbit (c.f. [46], [5], [27]). Ultimately, terminating alternans is a problem of controlling the timing of cell repolarization. By appropriately speeding up the repolarization of the long action potentials, the unstable period- 1 orbit can be stabilized to eliminate alternans in the system. In [59], using a newly developed methodology of isostable reduction in ODEs we were able to calculate infinitesimal isostable response curves (iIRCs) to determine the effect of small perturbations on individual 
cell repolarization timing. However, the control strategy previously developed in [59] lacked the framework necessary for application in connected cardiac tissue which is more relevant for in vivo applications. More recently, [60] presents the necessary framework for calculation of isostable reduction of partial differential equations, allowing for the reduction of PDEs which represent cardiac behavior. The aim of this paper is to develop strategies for elimination of cardiac alternans in PDE models of cardiac tissue. Ultimately, we find that such methods can eliminate alternans using significantly less energy than other comparable strategies and may have relevance in an experimental setting.

The organization of this paper is as follows: Section II gives details for the calculation of an isostable reduction on the PDE (1). Section III develops a control objective for eliminating alternans from the perspective of isostables and Section IV investigates the utility of a nearly optimal control strategy for achieving this control objective using voltage perturbations. Section V suggests a strategy for experimental calculation of isostable response curves, Section VI investigates control of alternans in tissue with two spatial dimensions, and concluding remarks are given in Section VII.

\section{ISOSTABLE REDUCTION}

Consider a weakly perturbed partial differential equation on the domain $\Omega$ :

$$
\frac{\partial}{\partial t} X(r, t)=F(X(r, t), r)+G(X(r, t))+p(r, t)
$$

where $X(r, t) \in \mathbb{R}^{m}$ represents the local state at the location $r \in \mathbb{R}^{d}$ at time $t, F(X(r, t), r) \in \mathbb{R}^{m}$ gives the local dynamics, $G(X(r, t))$ represents spatial coupling which could potentially be a function of derivatives of $X$, (e.g. diffusion), and $p(r, t)$ represents a weak external perturbation. In the absence of external perturbations, (i.e. $p(r, t)=0$ ) suppose the system eventually approaches stationary solution, denoted by $X_{o}$. Near the stationary solution, the dynamics behave according to

$$
X(r, t)-X_{0}=\sum_{j=1}^{\infty} s_{j}\left(X(r, 0)-X_{0}\right) \phi_{j} e^{\lambda_{j} t} .
$$

Here $\phi_{j}$ and $\lambda_{j}$ are eigenfunctions of eigenvalues of the system linearized about the stationary solution and ordered so that $\left|\lambda_{1}\right| \leq\left|\lambda_{2}\right| \leq \ldots$, and $s_{j}(X)$ give the coordinates of the state $X$ in the basis of eigenvectors.

This asymptotic behavior can be used to define a fully nonlinear isostable field $\psi=\Psi\{X(r)\}$ : [60], [36]

$$
\psi=\Psi\{X(r)\}=\frac{1}{\lambda_{1}} \log \left(\lim _{t \rightarrow \infty}\left[e^{-\lambda_{1} t} \int_{\Omega}\left(Z^{T}(r)\left(X(r, t)-X_{o}\right)\right) d \Omega\right]\right)+C .
$$

In (4) we take $X(r, 0)=X(r), Z(r) \in \mathbb{R}^{m}$ is a system observable, and ${ }^{T}$ indicates the transpose operator, and $C$ is a constant. When eigenvalues are distinct, the choice of $Z(r)$ has little bearing on the structure of isostables in the system provided it is not orthogonal to $\phi_{1}$ (c.f. [36]), but when $\lambda_{1}$ has geometric multiplicity greater than $1, Z(r)$ can be chosen as desired so that the resulting isostable field provides useful information about (2). Examining the individual components of (4), the exponential function in brackets grows at a rate determined by $\lambda_{1}$. The integral in equation (4) is proportional to $e^{\lambda_{1} t}$ so that the product of these two terms approaches a constant as time goes to infinity and gives a sense of the distance of the corresponding state to the stationary solution. Taking the logarithm and normalizing by $\lambda_{1}$ gives a coordinate system for which $d \psi / d t=1$ when $p(r, t) \equiv 0$ (see [59]), and the constant $C$ allows flexibility in defining $\psi=0$ to correspond to certain outward characteristics of a given trajectory (incorporated to make further computations easier as we will show in the sections to follow).

While the definition of isostables in (4) is rather technical, when applied to the cardiac PDE model (1), isostable coordinates have a more intuitive meaning. For example, let $Z(r)=\delta\left(r-r_{o}\right) \mathbb{1}$, where $\mathbb{1}$ is an appropriately sized vector of ones and $\delta$ is the delta function. With this choice, the integral from (4) only registers the dynamics at $r_{o}$ in the approach to the stationary solution. Suppose repolarization occurs at this location at $t=t_{0}$. Then, as $t-t_{0}$ increases both the diastolic interval (DI) and the isostable coordinates increase at the same rate (until the next action potential is elicited). Thus, for isostable coordinates defined in this way, larger values of isostables will correlate with larger values of DI which in turn produce larger action potential durations on the next beat. 
While (4) uniquely defines isostable coordinates for any state, direct calculation of an isostable field using (4) is generally of limited practical use because it requires computationally intensive simulation of (2) for each state $X(r)$ to estimate the approach to the stationary solution in infinite time. Often, we might only be interested in the isostable coordinates near a particular trajectory of interest, which we will denote by $\gamma$, and these coordinates can be defined in a more ad hoc manner by $X(r)=X(r, 0)$ with $X(r) \in \gamma$ forward in time, and choosing $\psi(X(r, t))=t$. Changing to isostable coordinates by taking the gradient of the isostable field using the chain rule yields

$$
\begin{aligned}
\frac{d \psi}{d t} & =\langle\nabla \psi(X), F(X(r, t), r)+G(X(r, t))+p(r, t)\rangle \\
& =1+\langle\nabla \psi(X), p(r, t)\rangle .
\end{aligned}
$$

Here, $\nabla \psi(X)$ is the infinitesimal isostable response curve (iIRC) which gives the system's response to an external perturbation, and $\langle\cdot, \cdot\rangle$ denotes the $L^{2}$ inner product. In the second line, we have used the fact that $d \psi / d t=1$ when $p(r, t) \equiv 0$ so that

$$
\langle\nabla \psi(X), F(X(r), r)+G(X)\rangle=1 .
$$

As detailed in [60], by defining $\nabla_{X(r, t)} \psi$ as the gradient of $\psi$ evaluated at $X(r, t)$ along $\gamma$, the iIRC can be calculated using

$$
\frac{\partial \nabla_{X(r, t)} \psi}{\partial t}=-J(X(r, t))^{\dagger} \nabla_{X(r, t)} \psi
$$

where $J \equiv \nabla[F(x(r, t), r)+G(X(r, t))]$, and ${ }^{\dagger}$ denotes the adjoint associated with the $L^{2}$ inner product. Practically, $J^{\dagger}$ must generally be estimated numerically by first discretizing (2) and solving for the adjoint of the resulting ODE system [28]. By approximating (5) as a linear system near the stationary solution, the iIRC can be calculated close to the stationary solution, and (7) can be solved by integrating backwards in time along $\gamma$ for the full nonlinear equations [60].

To cast (1) in the form of equation (2) so that we may calculate isostable response curves, let $X=$ $\left[V,\left[\mathrm{Ca}^{2+}\right]_{i},\left[\mathrm{Ca}^{2+}\right]_{s r}, f, d, m, h, j, f_{\mathrm{Ca}}, X_{\mathrm{Kr}}, X_{\mathrm{Ks}}, X_{\mathrm{to}}, Y_{\mathrm{to}}\right]^{T}$, consisting of all cellular state variables, and let $F(X(r, t))$ correspond to the differential equations for the cellular dynamics with a nominal parameter set given in [16]. Let $G(X(r, t))=D \frac{\partial^{2}}{\partial r^{2}} V(r, t)$, leaving $p(r, t)=\left[\begin{array}{llll}I_{\text {stim }} / C_{m} & 0 & \ldots & 0\end{array}\right]^{T}$. Consider an isostable model of this cardiac system:

$$
\begin{aligned}
\dot{\psi} & =1+\left\langle\mathcal{I}_{V}(r, \psi), I_{\text {stim }}(r, \psi)\right\rangle+\left\langle\mathcal{I}_{\left[\mathrm{Ca}^{2+}\right]_{i}}(r, \psi), 0\right\rangle+\cdots+\left\langle\mathcal{I}_{Y_{\mathrm{to}}}(r, \psi), 0\right\rangle \\
& =1+\left\langle\mathcal{I}(r, \psi), I_{\text {stim }}(r, \psi)\right\rangle .
\end{aligned}
$$

Note (with a slight abuse of notation) that the zero elements in first line of (8) must be compatible with the iIRCs. Because we can only perturb in the voltage direction, we define $\mathcal{I}(r, \psi) \equiv \mathcal{I}_{V}(r, \psi)$. In [60], we show that when $\gamma$ is close to the stationary solution, the iIRC corresponding to a given eigenfunction with a real eigenvalue is orthogonal to all other eigendirections and satisfies the normalization condition (6). For this problem, near the stationary solution, the eigenfunctions are difficult to determine numerically, and solely for the purposes of calculating the system's iIRC (all numerical simulations in the following sections use the unmodified dynamics), we make a few modifications which do not perceptibly alter the system dynamics. First, we reduce the dimensionality of the system by averaging [22], taking the slowly varying sarcoplasmic reticulum $\mathrm{Ca}^{2+}$ to be equal to a constant $318 \mu \mathrm{mol}$. Second, we modify the ionic current $I_{\mathrm{Kr}}$ (given in [16]) so that $I_{\mathrm{Kr}}=0$ when the variable $X_{\mathrm{Kr}}<10^{-4}$. With these modifications, when the system is close to the stationary solution, the value of $X_{\mathrm{Kr}}(r)$ (the variable associated with the gating variable for one of the potassium currents) approaches the stationary solution according to $X_{\mathrm{Kr}}(r)=\lambda_{1}\left(X_{\mathrm{Kr}}(r)-X_{\mathrm{Kr}, \infty}\right)$ with $X_{\mathrm{Kr}, \infty}$ denoting a steady state value of $X_{\mathrm{Kr}}$ and $\lambda_{1}$ denoting the slowest eigenvalue of the linearized system. Because the rate of approach of $X_{\mathrm{Kr}}(r)$ is the same at all locations $r$, there are an infinite number of eigenfunctions corresponding to the slowest direction of the stable manifold which span the function space $X_{\mathrm{Kr}}(r)$ ). These eigenfunctions span $X_{\mathrm{Kr}}(r)$ and are orthogonal to all other eigenfunctions with no component in $X_{\mathrm{Kr}}(r)$. Consider a cell at $r=r_{0}$. When the system is close to the stationary solution, any perturbation to $X_{\mathrm{Kr}}(r)$ for $r \neq r_{0}$ will have no effect on $X_{\mathrm{Kr}}\left(r_{0}\right)$ as it approaches the steady state. With this information, 
and using (6), near the stationary solution, the iIRC associated with a cell at $r_{0}$, which describes its infinite time approach towards the stationary solution, is given by

$$
\mathcal{I}_{\mathrm{X}_{\mathrm{Kr}}}(r, \psi)= \begin{cases}1 / \dot{X}_{\mathrm{Kr}} & \text { if } r=r_{0}, \\ 0 & \text { otherwise }\end{cases}
$$

An iIRC calculated using this initial condition describes the change in isostable for a cell at a given location $r_{0}$ caused by a small perturbation. Note that (9) is only valid near the stationary solution.

To numerically calculate the iIRC using (7), the trajectory $\gamma$ must approach the stationary solution so that (9) can be used to obtain an initial condition for (7). This can be accomplished, for instance, by taking $r_{0}=5 \mathrm{~cm}$, and locally setting $D=0$ between $r=0 \mathrm{~cm}$ and $r=10 \mathrm{~cm}$ at a moment immediately after the cell at $r_{0}$ fires. This allows the traveling waves to die out so that the system approaches the stationary solution. With an initial condition determined from (9), the iIRC at all other values in time is determined by integrating (7) backwards in time. As represented in Figure 2, the resulting iIRC is valid until $t_{D}$, the time that the cell at $r_{0}$ would have fired its next action potential. Note that in the control strategies to follow, alternans will be eliminated by perturbing the system on its way towards the stationary solution, but the traveling pulse will never die out.

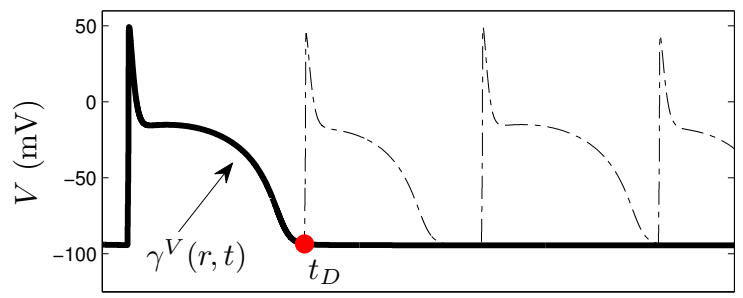

FIG. 2. In order to calculate the iIRC, $\gamma$ must come arbitrarily close to the stationary solution. By modifying the boundary conditions to allow the traveling wave to die out, we can numerically calculate an iIRC, which is accurate for the unmodified system until $t_{D}$. Here $\gamma^{V}(r, t)$ represents the voltage component of $\gamma$ at location $r$ along the ring.

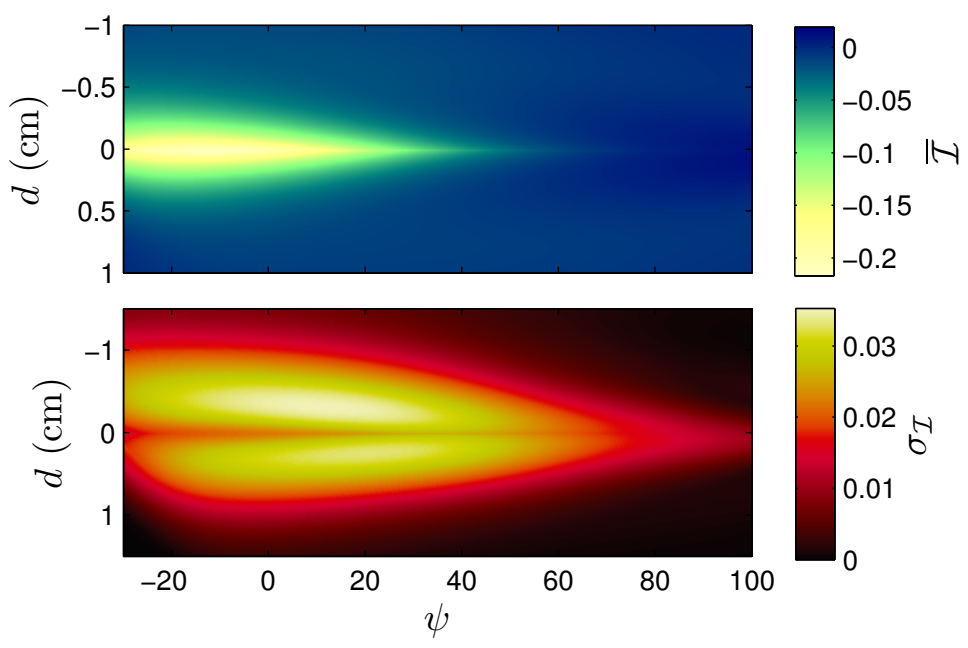

FIG. 3. Top and bottom panels show the mean and standard deviation of the iIRC, respectively, taken over 96 trials. Here $d$ is defined so that $\mathcal{I}(d, \psi)$ represents the effect of a perturbation on a cell $d \mathrm{~cm}$ away.

The system does not take the same trajectory $\gamma$ towards the stationary solution every time, and we calculate the iIRC 96 times using different initial conditions to determine an average $\operatorname{iRC}, \overline{\mathcal{I}}(r, \psi)$ shown in the top panel of Figure 3, with the associated standard deviation $\sigma_{\mathcal{I}}$ shown in the bottom panel. For the 
problem of eliminating alternans, each cell can be thought of as having its own isostable value and iIRC, but because the system is circularly symmetric, we simply report the iIRC in terms of the effect of a perturbation given at a signed distance $d$. A constant value can be added to the isostable field $\Psi\{X(r)\}$ without changing the property (6), and throughout the manuscript we will make use of this property as convenient. In Figure 3 , we shift the isostable coordinates so that $\psi=0$ corresponds to the time at which $\left.\frac{\partial V}{\partial t}\right|_{r=r_{0}}=0$, during a long action potential. The effect of an external stimulus is largest when $d$ is zero, corresponding to direct stimulation of that cell, and is near zero when $|d|>0.5 \mathrm{~cm}$. The standard deviation of the iIRC at $d \approx 0$ is small compared to the magnitude of the ilRC itself, allowing us to use $\overline{\mathcal{I}}(r, \psi)$ as an approximation of the true iIRC in the control strategy to follow.

\section{USING ISOSTABLES TO DEFINE A CONTROL OBJECTIVE TO ELIMINATE CARDIAC ALTERNANS}

From a dynamical systems perspective, alternans can be eliminated by driving the cell dynamics to an underlying unstable period-1 orbit [46], [5], [27]. In an experimental setting in which control nodes are sparsely distributed throughout the tissue, controlling the system to the unstable period-1 orbit is not a trivial task. For the following analysis, we consider a single cell isostable reduction throughout the ring, using methods similar to those of [59]. We assume the existence of an alternans-free state, $\psi_{0}(r, t)$, throughout the ring which evolves so that $\psi_{0}(r, t)=\psi_{0}(r-c t, 0)$, with $c>0$. Furthermore, we assume that the state at each location is close to the alternans-free trajectory, denoted by $\gamma_{a}+\mathcal{O}(\epsilon\|\Delta X\|)$, where $X$ represents the state variables of the system. This means that to leading order $\epsilon$, there is a 1 to 1 relationship between $\psi$ and the state variables.

Panel A of Figure 4 shows such a distribution, which can be found, for instance, by applying delayed feedback control methods [46], [42] to (1) until the control effort disappears. This distribution is an unstable solution to $(1)$ which is periodic in both space and time; the wavefront (located at $r \approx 4.6 \mathrm{~cm}$ in the figure) travels at approximately $57 \mathrm{~cm} / \mathrm{s}$ so that the period of oscillation is approximately $175 \mathrm{~ms}$. Isostables for each cell are scaled so that at all locations except the wavefront $\dot{\psi}=1$ in the absence of external stimuli. In this section, the constant $C$ from (4) is chosen so that $\psi=0$ corresponds to the time at which a cell has just repolarized (i.e. reached 95 percent of its resting potential). At the depolarizing wavefront, quiescent cells are excited again. The end goal of our control strategy is to guide the system to this unstable, alternans-free periodic orbit.

The local dynamics at location $r=x$ evolve according to (1):

$$
\left.C_{m} \frac{\partial V\left(\psi_{0}\right)}{\partial t}\right|_{r=x}=\left.D \frac{\partial^{2} V\left(\psi_{0}\right)}{\partial r^{2}}\right|_{r=x}-\left.I_{\text {ion }}\left(\psi_{0}\right)\right|_{r=x}+I_{\text {stim }}(x, t) .
$$

Through single grid point isostable reduction, provided the state dynamics are close to $\gamma_{a}$, we may write (10) as

$$
\left.\dot{\psi}\right|_{r=x}=1+\mathcal{I}_{s}\left(\left.\psi\right|_{r=x}\right) I_{\text {stim }}(x, t)
$$

Here, $\left.\psi\right|_{r=x}$ gives the isostable of the local dynamics at the location $r=x$, and $\mathcal{I}_{s}$ is the local isostable response curve. Note that in this reduction, both $\left.I_{\text {ion }}(\psi)\right|_{r=x}$ and $\left.D \frac{\partial^{2} V\left(\psi_{0}\right)}{\partial r^{2}}\right|_{r=x}$ guide the local dynamics along the trajectory $\gamma_{a}$, and therefore disappear in the reduction (11).

As derived in [59], we can calculate $\mathcal{I}_{s}(\psi)$ by solving an adjoint equation

$$
\frac{d \mathcal{I}_{s}}{d t}=-\nabla F^{T}(x(\psi)) \mathcal{I}_{s}
$$

Here, $\nabla F$ is the Jacobian, and $\nabla F^{T}(x(\psi))$ is the transpose, or adjoint, of the real-valued matrix $\nabla F(x(\psi))$, and $F(x)$ represents state dynamics of the transmembrane voltage and all auxiliary variables associated with $I_{\text {ion. }}$.

Suppose now that the distribution is not exactly at the ideal, $\psi_{0}$, but rather can be written as $\psi(r, t)=$ $\psi_{0}(r, t)+\epsilon \psi_{\epsilon}(r, t)$, with $0<\epsilon \ll 1$, so that $\psi_{\epsilon}$ represents a small perturbation from the ideal distribution. For clarity of notation in the following equation, we let $\left.P \equiv \psi_{\epsilon}\right|_{r=x}$, which is a constant with respect to 
derivatives in space. Suppose also that there are no external perturbations so that $I_{\text {stim }}=0$. To leading order, the dynamics at a given location $x$, starting with (10) can be written as

$$
\begin{aligned}
\left.C_{m} \frac{\partial V\left(\psi_{0}+\epsilon \psi_{\epsilon}\right)}{\partial t}\right|_{r=x} & =\left.D \frac{\partial^{2} V\left(\psi_{0}+\epsilon \psi_{\epsilon}+\epsilon P-\epsilon P\right)}{\partial r^{2}}\right|_{r=x}-\left.I_{\mathrm{ion}}\left(\psi_{0}+\epsilon \psi_{\epsilon}\right)\right|_{r=x} \\
& =\left.D \frac{\partial^{2}\left(V\left(\psi_{0}+\epsilon P\right)+\epsilon \frac{\partial V}{\partial \psi} \cdot\left(\psi_{\epsilon}-P\right)\right)}{\partial r^{2}}\right|_{r=x}-\left.I_{\mathrm{ion}}\left(\psi_{0}+\epsilon \psi_{\epsilon}\right)\right|_{r=x}+\mathcal{O}\left(\epsilon^{2}\right) \\
& =\left.D \frac{\partial^{2} V\left(\psi_{0}+\epsilon P\right)}{\partial r^{2}}\right|_{r=x}-\left.I_{\text {ion }}\left(\psi_{0}+\epsilon \psi_{\epsilon}\right)\right|_{r=x}+\left.\epsilon D \frac{\partial V}{\partial \psi} \cdot \frac{\partial^{2} \psi_{\epsilon}}{\partial r^{2}}\right|_{r=x}+\mathcal{O}\left(\epsilon^{2}\right) .
\end{aligned}
$$

Finally, equation (13) is in the same form as (10), with the term $\left.\epsilon D \frac{\partial V}{\partial \psi} \cdot \frac{\partial^{2} \psi_{\epsilon}}{\partial x^{2}}\right|_{r=x}$ taking the place of $I_{\text {stim }}(x, t)$. We may therefore rewrite (13) in the form of (11):

$$
\left.\left(\dot{\psi_{0}}+\epsilon \dot{\psi_{\epsilon}}\right)\right|_{r=x}=1+\left.\left(\mathcal{I}_{s}\left(\psi_{0}+\epsilon \psi_{\epsilon}\right) \epsilon D \frac{\partial V}{\partial \psi} \cdot \frac{\partial^{2} \psi_{\epsilon}}{\partial r^{2}}\right)\right|_{r=x}+\mathcal{O}\left(\epsilon^{2}\right)
$$

Finally, subtracting (11) with $I_{\text {stim }}=0$ from (14) and Taylor expanding the $\mathcal{I}_{s}$ in orders of $\epsilon$, we have

$$
\left.\epsilon \dot{\psi}_{\epsilon}\right|_{r=x}=\left.\left(\epsilon D \frac{\partial V}{\partial \psi} \cdot \mathcal{I}_{s}\left(\psi_{0}\right) \frac{\partial^{2} \psi_{\epsilon}}{\partial r^{2}}\right)\right|_{r=x}+\mathcal{O}\left(\epsilon^{2}\right)
$$

Equation (15) mandates that for all points at which the isostables are continuous in space, provided that $\left.\left(\epsilon D \frac{\partial V}{\partial \psi} \cdot \mathcal{I}_{s}\left(\psi_{0}\right)\right)\right|_{r=x}>0$, any local isostable gradient will spread through the system diffusively. Panels $\mathrm{C}$ and D of Figure 4 shows the term $\frac{\partial V}{\partial \psi}(\psi) \cdot \mathcal{I}_{s}\left(\psi_{0}\right)$ plotted as a function of $\psi$ for this system at two different scales, with the transmembrane voltage shown in panel B for reference. We find that this term is strictly positive, and is particularly large in magnitude soon after an action potential.
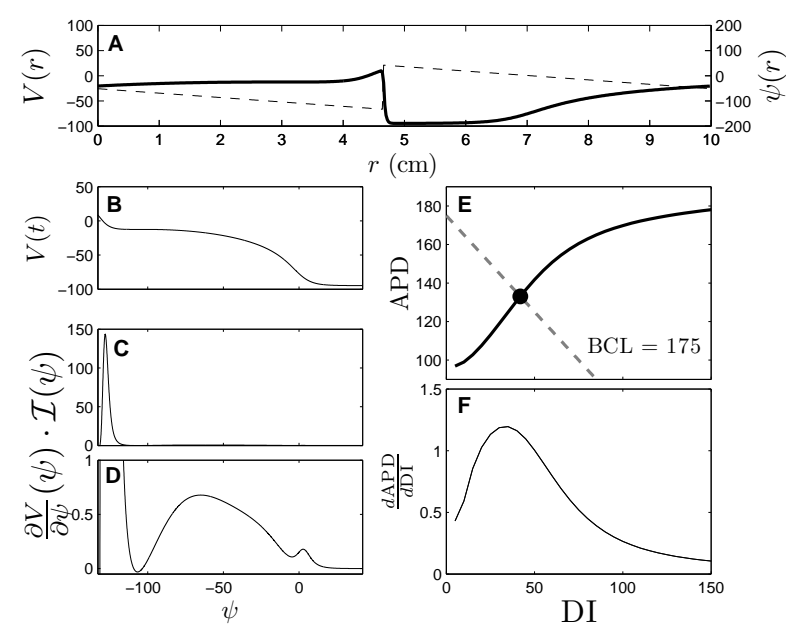

FIG. 4. Panel A shows an alternans-free solution of (1), and an underlying isostable distribution for each individual location throughout the network. The scales on the left and right correspond to the solid and dashed curve, respectively. Panels $\mathrm{C}$ and $\mathrm{D}$ show the term from equation (15) as a function of isostable, with the transmembrane voltage shown in panel B for reference. Because $\frac{\partial V}{\partial \psi}(\psi) \cdot \mathcal{I}_{s}(\psi)$ is predominantly greater than zero, $(15)$ shows that the isostable distribution $\psi_{\epsilon}$ should spread diffusively through the network. Panels $\mathrm{E}$ and $\mathrm{F}$ show the APD restitution curve as well as $d \mathrm{APD} / d \mathrm{DI}$. Here, the APD restitution curve is determined using an S1-S2 pacing protocol [31] with $\mathrm{S} 1=250 \mathrm{~ms}$. The BCL in this system is about $175 \mathrm{~ms}$, which gives an unstable fixed point at $\mathrm{DI}_{0} \approx 42 \mathrm{~ms}$.

Recall that (15) is only valid for points at which the isostable distribution is continuous in space. For the point at which this distribution is not continuous (i.e. at the wavefront) the dynamics can be reduced 
using the action potential duration (APD) restitution curve which gives the next action potential duration, defined to be the length of time the transmembrane voltage of a cell remains above resting potential after an action potential is fired, as a function of the DI, defined to be the amount of time the cell stays quiescent before the next action potential is fired:

$$
\mathrm{APD}_{i+1}=f\left(\mathrm{DI}_{i}\right)
$$

Here, because we have a series of action potentials, we refer to them with an index $i$. The APD restitution curve and its first derivative are shown in panels $\mathrm{E}$ and $\mathrm{F}$ of Figure 4, respectively. In this case, the basic cycle length (BCL), defined as the time between successive action potentials, is fixed, and determined by the wave speed $c$ and the length of the ring, and therefore BCL $=\mathrm{DI}+\mathrm{APD}$. One can show that (16) has a fixed point $\mathrm{DI}_{0}$ when $\mathrm{BCL}=f\left(\mathrm{DI}_{0}\right)+\mathrm{DI}_{0}$. As originally shown in [39], this fixed point will be unstable if $\left|\frac{d f}{d \mathrm{DI}}\left(\mathrm{DI}_{0}\right)\right|>1$, and this instability gives rise to the stable period-2 orbit responsible for alternans. We have strategically defined the isostables in this system so that positive and negative isostable values can be used interchangeably with DI and APD in equation (16), respectively. Therefore, we can write (c.f. [34])

$$
\psi_{\epsilon_{i+1}}(x)=-\Lambda \psi_{\epsilon_{i}}(x)
$$

where $\Lambda=\frac{d f}{d \mathrm{DI}}\left(\mathrm{DI}_{0}\right)$, and $\psi_{\epsilon_{i}}(x)$ and $\psi_{\epsilon_{i+1}}(x)$ denote the value of $\psi_{\epsilon}$ at location $r=x$ before and after a given action potential. Equation (15) and (17) specify a reduced system. The diffusive element of the system (15) will tend to bring it towards $\psi_{\epsilon}(r)=\bar{\psi}_{\epsilon}$, the average value of the initial distribution, and equation (17) describes the inherent instability of the local dynamics. Therefore, our control strategy will be to actively drive the system to $\psi_{0}(r)+\epsilon \psi_{\epsilon}(r)$ where $\bar{\psi}_{\epsilon}=0$. Provided that the diffusion acts much faster than the inherent instability of the local dynamics, the system will settle close to $\psi_{0}$. If the diffusion does not act quickly enough, we can apply the external control a few cycles later in order to gradually drive the system to $\psi_{0}$.

\section{AN OPTIMAL CONTROL STRATEGY FOR ELIMINATING ALTERNANS}

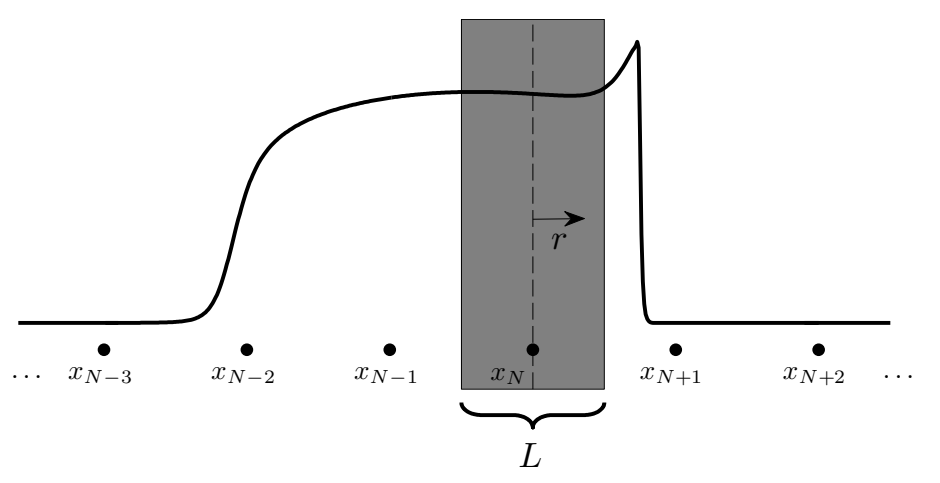

FIG. 5. The reduced dynamics on the one-dimensional domain are described by equation (19).

Suppose that we have $2 M+1$ control nodes, equally spaced at a distance $L$. Let $u_{N}(t)$ represent the control applied at node $N$ which is of order $\epsilon$. Using the coordinate system presented in Figure 5 , the local reduced dynamics at position $r$, which we denote by $\psi_{r}$, are

$$
\dot{\psi}_{r}=1+\sum_{j=-M}^{M} u_{N+j}(t) \mathcal{I}\left(j L-r, \psi_{r}\right)+\mathcal{O}\left(\epsilon^{2}\right) .
$$

Here, the summation represents the effect of input from each control node. Recall from Figure 3 that $\mathcal{I}(d, \psi)$ is approximately zero when $|d|$ is sufficiently large. We therefore define $\beta^{+}$(resp. $\beta^{-}$) as the smallest positive 
integer (resp. largest negative integer) such that $\mathcal{I}\left(\left(\beta^{+}+1\right) L-r, \psi_{r}\right)=\mathcal{O}(\epsilon)\left(\operatorname{resp} . \mathcal{I}\left(\left(\beta^{-}-1\right) L-r, \psi_{r}\right)=\mathcal{O}(\epsilon)\right)$ for every $r \in[-L / 2, L / 2]$ and for all values of $\psi_{r}$. This definition allows us to rewrite (18) as,

$$
\dot{\psi}_{r}=1+\sum_{j=\beta^{-}}^{\beta^{+}} u_{N+j}(t) \mathcal{I}\left(j L-r, \psi_{r}\right)+\mathcal{O}\left(\epsilon^{2}\right), \quad \text { for every } r \in[-L / 2, L / 2] .
$$

Suppose that in the region which contains electrodes which have a larger than $\mathcal{O}\left(\epsilon^{2}\right)$ effect on the isostable coordinates, the severity of alternans is similar (i.e. $\mathrm{APD}_{n}-\mathrm{APD}_{n-1}=\alpha+\mathcal{O}(\epsilon)$ ). Suppose also that the wave speed in this region of tissue is equal to $c+\mathcal{O}(\epsilon)$. This means that if $\psi_{r}=0$ at $t=0$, then $\psi_{r+d}$ will reach zero at $t=d / c+\mathcal{O}(\epsilon)$.

We assume that each node acts independently, and require that an $\mathcal{O}(\epsilon)$ change in the severity of alternans will lead to an $\mathcal{O}(\epsilon)$ change in the applied control. In this section, it will be convenient to shift the isostable coordinates so that $\psi_{r_{0}}=0$ corresponds to the time at which $\left.\frac{\partial V}{\partial t}\right|_{r=r_{0}}=0$, during a long action potential (identical to the shift from Section II). We also assume that each node will apply control which lasts for a duration of $k \mathrm{~ms}$, starting when the isostable at that node reaches $\psi=0$. Under these assumptions, to leading order $\epsilon$ the external control applied at each node will be identical in shape, but time shifted by the time it takes for the wave to travel between control nodes. This allows us to rewrite (19) as

$$
\dot{\psi}_{r}=1+\sum_{j=\beta^{-}}^{\beta^{+}} u(t-j L / c) \mathcal{I}\left(j L-r, \psi_{r}\right)+\mathcal{O}\left(\epsilon^{2}\right), \quad \text { for every } r \in[-L / 2, L / 2],
$$

where $u(z)=0$ when either $z<0$ or $z>k$. Asymptotically expanding $\psi_{r}$ in powers of $\epsilon$,

$$
\psi_{r}(t)=\psi_{r}^{(0)}(t)+\epsilon \psi_{r}^{(1)}(t)+\epsilon^{2} \psi_{r}^{(2)}(t),
$$

we find that $\psi_{r}^{(0)}(t)=\psi_{r}(0)+t$. We will define $t=0$ to be the time at which $\psi_{0}=0$, which can be written more concisely as $\psi_{0}(0)=0$. Therefore $\psi_{r}(0)=-r / c$. Substituting this result into (20) yields

$$
\dot{\psi}_{r}=1+\sum_{j=\beta^{-}}^{\beta^{+}} u(t-j L / c) \mathcal{I}(j L-r, t-r / c)+\mathcal{O}\left(\epsilon^{2}\right), \quad \text { for every } r \in[-L / 2, L / 2] .
$$

Recall that our goal is to drive the average of the isostable distribution in the shaded region of interest in Figure 5 to the unstable period-1 orbit. Taking the spatial average of (22) yields

$$
\dot{\bar{\psi}}=\frac{1}{L} \int_{-L / 2}^{L / 2} \dot{\psi}_{r} d r=1+\frac{1}{L} \int_{-L / 2}^{L / 2}\left[\sum_{j=\beta^{-}}^{\beta^{+}} u(t-j L / c) \mathcal{I}(j L-r, t-r / c)\right] d r+\mathcal{O}\left(\epsilon^{2}\right),
$$

where $\dot{\bar{\psi}}$ is the average rate of change in isostables in the shaded region.

We can use (23) as part of a strategy to eliminate alternans. Suppose that the next action potentials will be fired $T \mathrm{~ms}$ after the present action potentials. Then, we may guide the system from the stable period- 2 orbit to the unstable period-1 orbit by requiring (c.f. [59])

$$
\int_{0}^{T} \dot{\bar{\psi}} d t=T-\zeta\left(\mathrm{APD}_{n}-\mathrm{APD}_{n-1}\right) / 2
$$

which implies the following boundary condition

$$
\int_{0}^{T}(\dot{\bar{\psi}}-1) d t=-\zeta\left(\mathrm{APD}_{n}-\mathrm{APD}_{n-1}\right) / 2,
$$

where $\zeta$ is a positive constant. Recall that we apply control when the cells are currently firing long action potentials, so that $\mathrm{APD}_{n}-\mathrm{APD}_{n-1}<0$. In equation (25), setting $\zeta=0$ would mean that at the next action potential, control would never be applied, and the next action potentials will follow a short action potential 
trajectory. If $\zeta=2$, the result of the control would be to speed up the isostable dynamics so that on average, the cells would take another long APD path for the next action potential. Intuitively, assuming that the distribution of isostables along the unstable alternans free trajectory lies between these two extremes, some $\zeta \in(0,2)$ will yield a successful control strategy (c.f. [5]).

Recall that for $t<0$ and $t>k, u(t)=0$. This allows us to conveniently time shift the terms in the integrand of (25) after substituting (23):

$$
\int_{0}^{T}(\dot{\bar{\psi}}-1) d t=\int_{0}^{k}\left[\frac{1}{L} \int_{-L / 2}^{L / 2}\left[\sum_{j=\beta^{-}}^{\beta^{+}} u(t) \mathcal{I}(j L-r, t-r / c+j L / c)\right] d r\right] d t .
$$

For convenience of notation, let $f(t)=\frac{1}{L} \int_{-L / 2}^{L / 2}\left[\sum_{j=\beta^{-}}^{\beta^{+}} \mathcal{I}(j L-r, t-r / c+j L / c)\right] d r$. By defining an auxiliary equation $\dot{\Psi} \equiv u(t) f(t)$, we can cast this as an optimal control problem by minimizing the cost functional $\mathcal{M}[u(t)]=\int_{0}^{k} u^{2}(t) d t$, which gives the power associated with the stimulus, and can apply calculus of variations to minimize [30]

$$
C[u(t)]=\int_{0}^{k}\left[u^{2}(t)+\lambda(\dot{\Psi}-u(t) f(t))\right] d t,
$$

where $\lambda$ is a Lagrange multiplier. The resulting Euler-Lagrange equations are

$$
\begin{aligned}
u(t) & =\lambda f(t) / 2, \\
\dot{\Psi} & =\lambda f^{2}(t) / 2, \\
\dot{\lambda} & =0 .
\end{aligned}
$$

The optimal control $u^{*}(t)$ can be found by solving (29) and (30) subject to $\Psi(0)=0$ and $\Psi(k)=-\zeta\left(\operatorname{APD}_{n}-\right.$ $\left.\mathrm{APD}_{n-1}\right) / 2$. By noting that (30) requires $\lambda$ to be a constant, and integrating (29) directly, we can explicitly solve for the required control as a function of time:

$$
u^{*}(t)=\frac{-\zeta\left(\mathrm{APD}_{n}-\mathrm{APD}_{n-1}\right) f(t)}{2 \int_{0}^{k} f^{2}(\tau) d \tau}
$$

Thus, to leading order $\epsilon$, the optimal control is proportional to a superposition of appropriately time shifted iIRCs. The PDE (1) is rather unwieldy and directly calculating an optimal control for elimination of alternans would not be possible. However, isostable reduction allows for the derivation of the relatively simple control scheme (31). Panel A of Figure 6 shows the calculated optimal control $u(t)$ when $k=70$ and $\zeta=1.3$, scaled by the severity of alternans. Perhaps not surprisingly, when the nodes are spaced closer together, the required control at each node is smaller in magnitude. For comparison, we also test the non-optimal control strategy presented in [46]:

$$
u(t)= \begin{cases}\nu(V(t)-V(t-\delta)) & \text { if } V(t)-V(t-\delta)<0, \\ 0 & \text { otherwise }\end{cases}
$$

where $\nu$ is a positive constant and $\delta$ is the time between successive depolarizations of a single cell. Intuitively, (32) works similarly to (31) by providing a hyperpolarizing stimulus when a region of tissue has a long alternan, but also requires simultaneous accurate monitoring of the transmembrane potential.

We test each control strategy on (1) with independent and identically distributed zero mean, unit intensity white noise added to the voltage variable. For each control strategy, more power is initially consumed because the controller is driving the system to the period-1, alternans-free orbit (see $t=1$ to $t=2$ in panel C). After this initial period of time, the controller requires considerably less energy $\left(E=\sum_{j} \int u_{j}^{2}(t) d t\right)$ to maintain the alternans-free state, making small adjustments to combat the effects of noise. Average power is reported as average energy required per second to maintain the alternans-free state. The approximately optimal control strategy uses 2 to 4.5 times less energy than the non-optimal strategy. Results for choices of $\zeta \in[1.1,1.5]$ are not significantly different. 

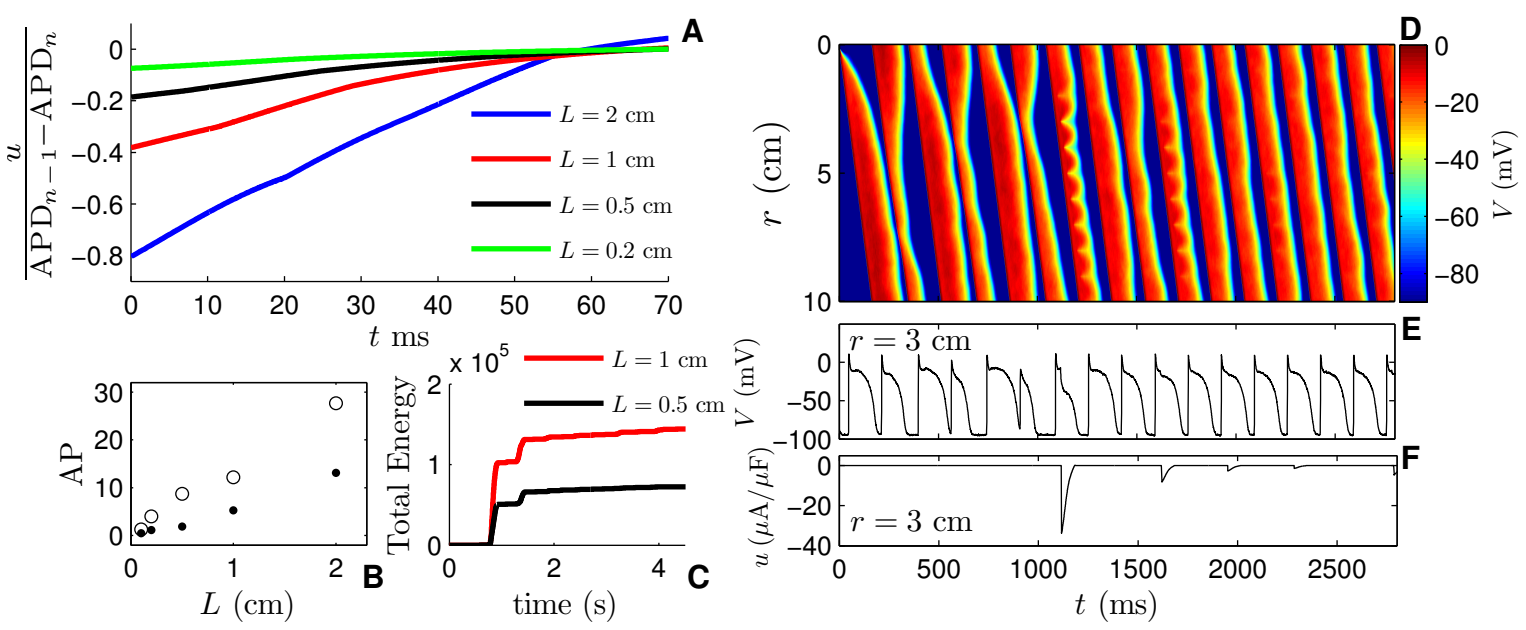

FIG. 6. Using the iIRC, optimal stimuli to eliminate alternans calculated from (31) for $k=70$ and $\zeta=1.3$ and different node spacings $L$, are shown in panel A with $u$ given in units of $\mu \mathrm{A} / \mu \mathrm{F}$ and APDs in ms. Panel B compares the average power (AP) once the controller reaches a steady state, for the optimal strategy (dots) and the non-optimal strategy from (32) (open circles). At each spacing, the approximately optimal strategy uses between 2 and 4.5 times less energy than the non-optimal strategy. Panel $\mathrm{C}$ shows the total cumulative energy consumption as a function of time for two different node spacings. Panel D shows the spatial transmembrane voltage as a function of time with $L=1 \mathrm{~cm}$. Alternans can be seen in the tissue when $t<1000 \mathrm{~ms}$, and are quickly eliminated when the controller is turned on. Panels $\mathrm{E}$ and $\mathrm{F}$ show the transmembrane voltage and control effort at $r=3 \mathrm{~cm}$.

\section{PROPOSED EXPERIMENTAL MEASUREMENT OF IIRCS}

In the previous example, the optimal control strategy requires a reasonably accurate estimate of the iIRC. If a good approximation of the model is known, an iIRC could be calculated using the adjoint equation (7). However, given the complicated nature of (1) it may be more accurate to measure the iIRC using strategies akin to the direct method [26], [37], a well established technique for experimentally estimating phase response curves in periodically oscillating systems. In oscillatory systems, the direct method is implemented by applying a perturbation when the system is at a known phase of oscillation allowing for a single measurement of the phase response curve obtained by calculating the change in phase due to the perturbation [1], [2], [40]. Multiple measurements at different phases can be taken to estimate the entire phase response curve. While each of these measurements depends on factors such as pacing history [53], stimulus amplitude and duration [41], and noise [12], they generally give a good understanding of the perturbed dynamics and are useful for predicting behavior in living systems [57], [29].

Here, we detail how a direct method could be used to experimentally implement the control strategy from the previous section in, e.g., Purkinje fibers [5], [32] which have been used to validate alternans control strategies in one-dimensional tissue. Our approach follows from the interpretation of the iIRC, $\mathcal{I}(d, \psi)$, as the change in the isostable coordinate resulting from a perturbation at a distance $d$, with $\psi$ being the isostable coordinate when the stimulus was applied.

Recall that from the definition (4) isostable coordinates give a sense of a system's approach to a stationary solution. For this reason, measuring differences in the onset of action potentials (the basis for the direct method in applications with periodic trajectories) does not provide information about the isostable coordinates. Instead, one can gauge how a perturbation affects the isostable using the APD restitution curve [14], which gives the duration of the next action potential as a function of the time since a cell has been quiescent (also known as the recovery time or diastolic interval). Generally, longer recovery times will lead longer action potentials. Here it will be convenient to scale and shift the isostable coordinates so that $\psi=0$ corresponds to the time at which a cell repolarizes (i.e. reaches 95 percent of its resting potential) with $\dot{\psi}=1$ in the absence of forcing so that

$$
\mathrm{APD}=\kappa(\psi)
$$


Note here that the constant shift of the isostable coordinates is different than it was in Section II. The APD restitution curve for this system is shown in panel $\mathrm{B}$ of Figure 7 . For a constant pacing rate $P$ that does not produce alternans, the system will settle to a state for which the action potentials are constant on a beat to beat basis which can be found by solving the equation $P=\psi+\kappa(\psi)=\psi^{*}+$ APD $^{*}$ [14], where $\mathrm{APD}^{*}=\kappa\left(\psi^{*}\right)$. For a given voltage perturbation, using Taylor expansion, the effective change in isostable can be determined by measuring the next action potential duration according to

$$
\frac{\Delta \mathrm{APD}}{\kappa^{\prime}\left(\psi^{*}\right)}=\Delta \psi+\mathcal{O}\left(\Delta \psi^{2}\right)
$$

where $\triangle \mathrm{APD}=\mathrm{APD}^{+}-\mathrm{APD}^{*}$, with $\mathrm{APD}^{+}$being the duration of the action potential following the perturbation (see panel A of Figure 7).

Suppose instead of a ring, we have a fiber (1) with no-flux boundary conditions. With multiple recording nodes and a single stimulating electrode at one end the following protocol can be used to experimentally determine the iIRC: 1) At one end of the fiber, pace at a constant rate $P$ chosen so that alternans do not develop in the system. 2) After the APDs are close to the steady state value APD*, apply a short pulsatile perturbation at time $t_{p}$ with strength $u$ and duration $\Delta t$ with the stimulating electrode. 3 ) For each recording node at a distance $d$ from the perturbing electrode, let $\psi_{p} \equiv t_{p}-t_{s}-$ APD $^{*}$ with $t_{s}$ defined to be the time at which the recorded cell spiked before the perturbation was applied. The value of $\Delta \psi$ can be determined from (34) by measuring the duration of the next action potential. 4) At each recording electrode obtain a data point $\mathcal{I}_{V}\left(d, \psi_{p}\right)=\frac{\Delta \psi}{u \Delta t}$. 5) Repeat steps 2-4 until a $\mathcal{I}_{V}(r, \psi)$ can be obtained with a fit to the data. Panel $\mathrm{C}$ of Figure 7 shows a polynomial fit to the data obtained using the above procedure with white noise
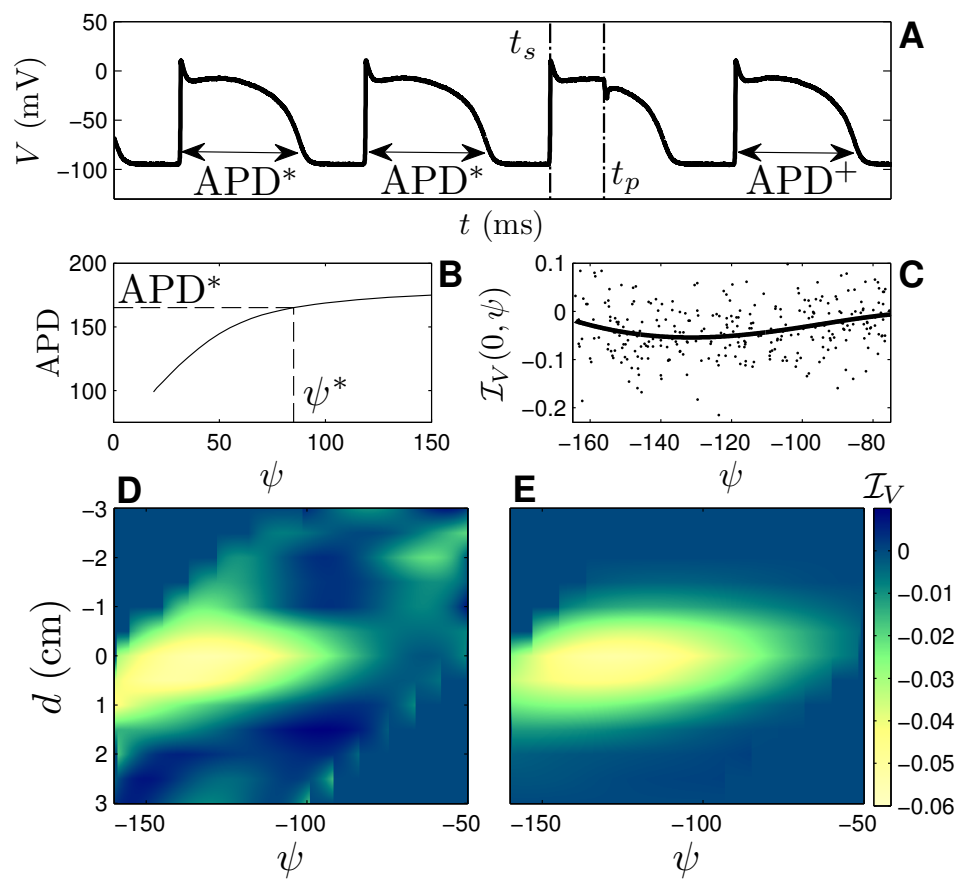

FIG. 7. In panel A, once the APDs reach their steady state value, a perturbation is given. $\mathrm{APD}^{+}$is measured as on the following action potential. Panel B shows the APD restitution curve for this system using an S1-S2 pacing protocol [31] with $S 1=250 \mathrm{~ms}$. Panel C shows individual datapoints for the iIRC obtained from (34) fitted to a polynomial (black line). Panels D and E show the resulting ilRCs using data from a noisy and noiseless system, respectively.

of intensity 0.2 added to the transmembrane voltage equation (1) to model system noise and measurement error. Each data point of the iIRC is calculated with a hyperpolarizing perturbation of $u=-30 \mu \mathrm{A} / \mu F$ applied for $\Delta t=4 \mathrm{~ms}$. Panel D shows an iIRC interpolated using data from recording nodes spaced $0.5 \mathrm{~cm}$ apart. Panel E shows an iIRC determined without noise in the system. In both cases the obtained iIRC is 
similar to the one obtained with the adjoint equation (7), but is less concentrated around $d=0$. Because we assumed that we only have one stimulating electrode, perturbation timing was chosen to give the best measurement of the system near $d=0$ and no data points in the top-left and bottom-right corners were obtained.

Using the iIRCs obtained from the noisy and noiseless data, we can apply the control strategy (31) to eliminate alternans. In this application, we take $t=0$ at each stimulator to be the time at which $\psi \approx-130$ during a long action potential, estimated by assuming that when a cell fires a long action potential, $\psi=-\mathrm{APD}_{L}^{-}$, where $\mathrm{APD}_{L}^{-}$is the duration of the previous long action potential. The stimuli obtained from the noiseless (resp. noisy) data are shown in the top left (resp. bottom left) panel of Figure 8 with $\zeta=1.3$. They are qualitatively similar to the stimuli obtained using the numerically obtained iIRC from Figure 3. In an identical numerical test as the one shown in Figure 6, each applied control strategy quickly eliminates alternans in the system. Using the stimuli associated with the iIRC obtained using the direct method requires slightly less power than the stimuli associated with the iIRC calculated numerically using (7). An iIRC obtained from a noisy system using the direct method only leads to a slight increase in overall power usage.

In an experimental setting iIRCs obtained from the direct method give the change to APDs themselves in response to a perturbation. When the iIRCs are obtained numerically using (7), they give the change to the approach along the slowest direction of the stable manifold from a perturbation. Because the control strategy itself uses the APDs as an indicator of the severity of alternans, this may explain why experimental iIRCs produce stimuli which are slightly more efficient. As a final note, measurement of the iIRC using the direct method requires knowledge of the APD restitution curve, which may not be available in an experimental setting. If this is the case, simply assuming $f^{\prime}\left(\psi^{*}\right)=1$ in equation (34) will yield stimuli for which the shape is correct but the magnitude is potentially wrong. The appropriate stimulus could be found, for instance, by applying the resulting control and modifying the magnitude until alternans are eliminated.
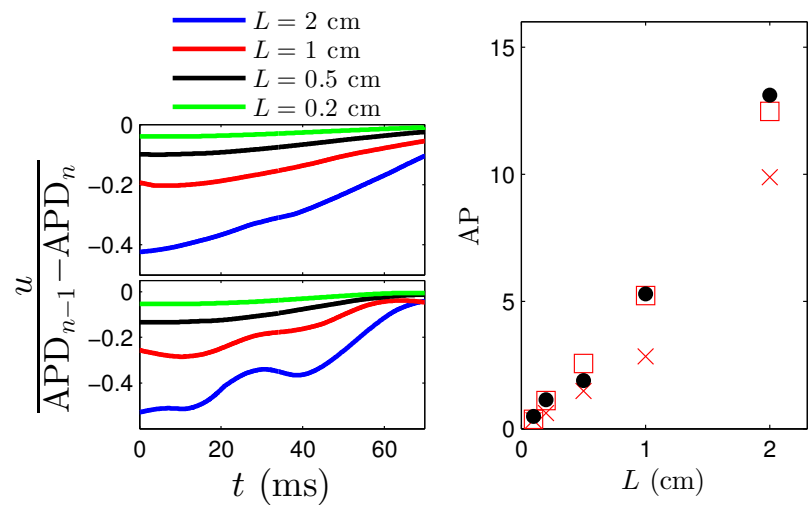

FIG. 8. Top-left and bottom-left panels show the optimal stimuli obtained from iIRCs using the direct method from a noiseless and noisy system (1), respectively. The right panel shows the average power required to maintain the alternans-free state for different node spacings $L$. Stimuli are obtained using an iIRC inferred from noisy data (red squares), noiseless data $($ red $\times$ ), and also calculated exactly using the adjoint equation (7) (black dot)

\section{TWO-DIMENSIONAL REDUCTION AND CONTROL OF ALTERNANS}

The isostable reduction strategies described in the previous sections extend naturally to higher dimensions; here we show that analogous alternans elimination strategies could be implemented in tissue with higher spatial dimension. To begin, we will assume that we have a $4 \mathrm{~cm}$ square patch of cardiac tissue:

$$
\begin{aligned}
C_{m} \frac{\partial V(x, y, t)}{\partial t}= & D \Delta V(x, y, t) \\
& +\left(-I_{\text {ion }}(x, y)+I_{\text {stim }}(x, y, t)+I_{\text {pacemaker }}(x, y, t)\right) .
\end{aligned}
$$


Here, $x$ and $y$ represent two-dimensional spatial coordinates, boundary conditions are given by $\partial V / \partial \eta=0$ where $\eta$ is a vector normal to the boundary (i.e. no-flux), $\Delta$ denotes the Laplacian, $I_{\text {pacemaker }}$ is a stimulating current included to elicit action potentials on one side of the domain at regular intervals, and all other variables and model parameters are taken identically to those in (1). We take $I_{\text {pacemaker }}$ to inject $100 \mu \mathrm{A} / \mu \mathrm{F}$ pulses of current at $\Delta t$ ms intervals along the side for which $x=0$. In a noiseless setting, this results in a linear wave front which propagates through the tissue. Here, we are modeling isotropic tissue, but note that it would be straightforward to calculate iIRCs incorporating tissue anisotropy in tissue conductivity [6]. A square lattice of electrical simulators with spacing $L$ used to provide $I_{\text {stim }}$ is arranged so that the wave front reaches each row at the same time (see panel A of Figure (9)). The numerical methods for calculating iIRCs represented by equation (2)-(9) extend straightforwardly to a two-dimensional setting. Similar to the protocol used for calculating iIRCs in one spatial dimension, we shift the isostable coordinates so that $\psi_{x_{o}, y_{o}}=0$ corresponds to the time at which $\left.\frac{\partial V}{\partial t}\right|_{(x, y)=\left(x_{o}, y_{o}\right)}=0$ during a long action potential. Setting $I_{\text {stim }}=0$ for the remainder of the simulation, we allow the system to approach the stationary solution in order to determine an initial condition for (7), and simulate (7) in backward time to determine the iIRC in the fully nonlinear regime. This strategy is used to calculate the iIRCs with respect to 49 different excitations of the domain. Pulsing rates between 160 and $210 \mathrm{~ms}$ are used in order to determine an average iIRC for voltage perturbations with respect to the location $\left(x_{o}, y_{o}\right)=(2,2)$. Results are reported in panels B-E of Figure 9 as $\overline{\mathcal{I}}\left(d_{x}, d_{y}, \psi\right)$ where $\overline{\mathcal{I}}$ is the averaged iIRC and $\left(d_{x}, d_{y}\right)=\left(x-x_{o}, y-y_{o}\right)$. Similar to the onedimensional calculations, perturbations do not greatly alter the isostable coordinate of any locations farther than about $0.8 \mathrm{~cm}$ away.

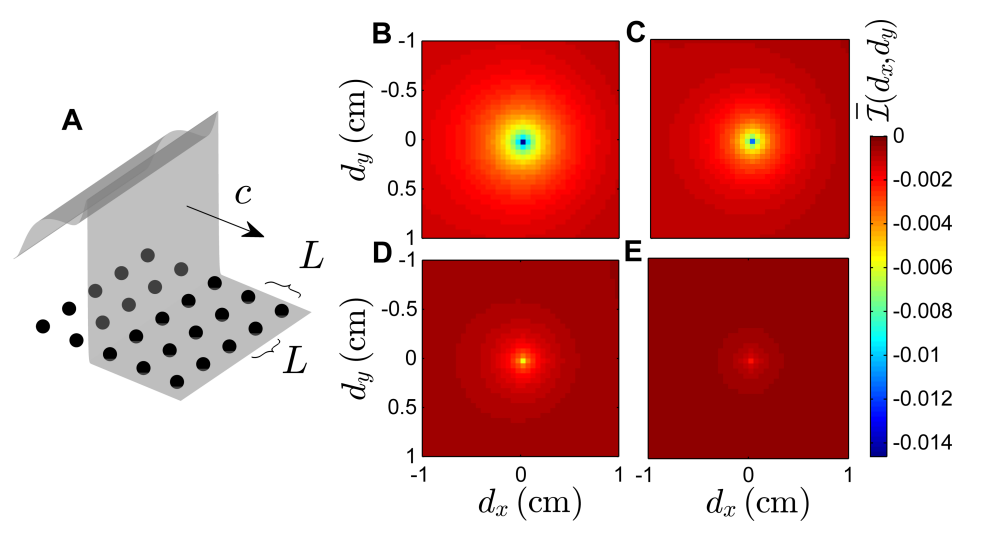

FIG. 9. Panel A gives a schematic of the two-dimensional setup. The wavefront propagates through the domain at speed $c$. A square lattice of stimulators is placed so that $L$ is the vertical and horizontal distance between successive stimulators. Panels B, C, D, and E give an averaged iIRC calculated in the two-dimensional tissue at $\psi=0,20,40$. and 60 .

The analysis from Section III can be repeated for the two-dimensional domain to justify a control strategy for elimination of alternans which drives the system to a state $\psi_{0}(x, y)+\epsilon \psi_{\epsilon}(x, y)$ where $\psi_{0}(x, y)$ represents the alternans-free state, $\epsilon$ is a small positive constant, and $\int_{y} \int_{x} \psi_{\epsilon} d x d y=0$. In the periodic, one-dimensional case, we were able to develop a control strategy to achieve this control goal everywhere on the domain. Here, we will show that a similar strategy can be used to achieve this control objective at locations away from the boundary of the domain. Numerical simulations presented later in this section show that this strategy is sufficient for elimination of alternans in (35) and does so using orders of magnitude less energy than comparable methods.

To begin, consider a stimulator located at $(x, y)=\left(x_{1}, y_{1}\right)$ with associated control signal $u_{0,0}(t)$. Also, let $u_{i, j}$ denote the input from a stimulator located at $(x, y)=\left(x_{1}+i L, y_{1}+j L\right)$. Here, $i$ and $j$ are allowed to be negative. Analogous to the setup in the one-dimensional case, Figure 10 highlights a square patch of length $L$ centered about the stimulation $u_{0,0}$ for which we will design a strategy to control the isostable dynamics. A shifted coordinate system, $r_{x}=x-x_{1}$ and $r_{y}=y-y_{1}$ allows us to write the perturbed isostable dynamics 
of any location in this patch:

$$
\dot{\psi}_{r_{x}, r_{y}}=1+\sum_{j=-n_{y}}^{p_{y}} \sum_{i=-n_{x}}^{p_{x}} u_{i, j}(t) \mathcal{I}\left(i L-r_{x}, j L-r_{y}, \psi_{r_{x}, r_{y}}\right)+\mathcal{O}\left(\epsilon^{2}\right)
$$

Here, $\psi_{r_{x}, r_{y}}$ represents the isostable reduced coordinates at the spatial position $\left(r_{x}, r_{y}\right), \mathcal{I}\left(d_{x}, d_{y}, \psi\right)$ is the iIRC, and indices $n_{x}$ and $p_{x}$ (resp. $n_{y}$ and $p_{y}$ ) represent the number of stimulators which extend in the negative and positive $x$ direction (resp. $y$ direction). Equation (36) represents the summation of the input from each control node and is analogous to (19) from the one-dimensional case. Because the two-dimensional domain is homogeneous we take the iIRC to be identical to $\overline{\mathcal{I}}\left(d_{x}, d_{y}, \psi\right)$, the average calculated iIRC shown in Figure 9. This approximation will be most accurate at locations far from the boundaries of the domain.

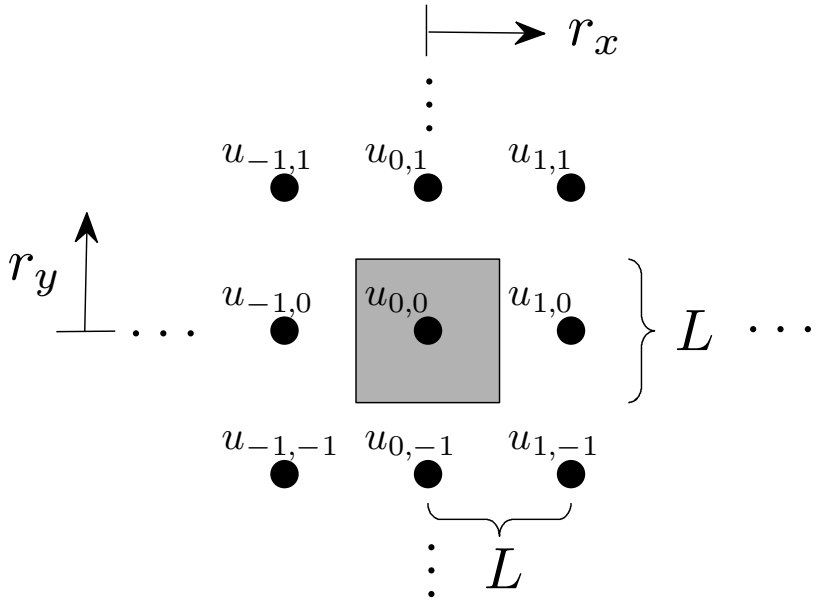

FIG. 10. The control strategy is designed to modulate the isostable dynamics in a square patch of tissue centered about a given stimulator. Ultimately, because the tissue is assumed to be homogeneous, the control goal of eliminating alternans in a square patch around a stimulator in the center of the tissue will also eliminate alternans in all locations far from the tissue boundary.

Mirroring the strategy from in Section IV, we can first notice that $\mathcal{I}\left(d_{x}, d_{y}, \psi\right)$ is small for sufficiently large values of $\left|d_{x}\right|$ and $\left|d_{x}\right|$. Therefore, for large values of $|i|$ and $|j|, \mathcal{I}\left(i L-r_{x}, j L-r_{y}, \psi_{r_{x}, r_{y}}\right)$ will be an $\mathcal{O}\left(\epsilon^{2}\right)$ term for every $r_{x}, r_{y} \in[-L / 2, L / 2]$. We can then define $\beta_{y}^{-} \leq n_{y}, \beta_{x}^{-} \leq n_{x}, \beta_{y}^{+} \leq p_{y}, \beta_{x}^{+} \leq p_{x}$ such that

$$
\dot{\psi}_{r_{x}, r_{y}}=1+\sum_{j=-\beta_{y}^{-}}^{\beta_{y}^{+}} \sum_{i=-\beta_{x}^{-}}^{\beta_{x}^{+}} u_{i, j}(t) \mathcal{I}\left(i L-r_{x}, j L-r_{y}, \psi_{r_{x}, r_{y}}\right)+\mathcal{O}\left(\epsilon^{2}\right), \quad \text { for every } r_{x}, r_{y} \in[-L / 2, L / 2] .
$$

In the region which contains electrodes which have a larger than an $\mathcal{O}\left(\epsilon^{2}\right)$ effect on the isostable coordinates, we assume that the severity of alternans is similar (i.e. $\mathrm{APD}_{n}-\mathrm{APD}_{n-1}=\alpha+\mathcal{O}(\epsilon)$ ), and the wave speed in the tissue is equal to $c+\mathcal{O}(\epsilon)$ where $c$ is a constant. We require that each node acts independently, and each control application lasts $k \mathrm{~ms}$ and begins when the isostable at the given control node reaches $\psi=0$. We also explicitly require that an $\mathcal{O}(\epsilon)$ change in the severity of alternans leading to an $\mathcal{O}(\epsilon)$ change in the applied control. As in Section IV, to leading order $\epsilon$, the control at each node is identical in shape and time shifted by the time it takes to travel between each node. Because the wave progresses at a constant rate in the $x$ direction, equation (37) becomes

$$
\begin{gathered}
\dot{\psi}_{r_{x}, r_{y}}=1+\sum_{\substack{j=-\beta_{y}^{-} \\
\text {for every }}}^{\beta_{x}, r_{y} \in\left[-\beta_{x}^{-}\right.} \sum^{\beta_{x}^{+}} u(t-i L / c) \mathcal{I}\left(i L-r_{x}, j L-r_{y}, \psi_{r_{x}, r_{y}}\right)+\mathcal{O}\left(\epsilon^{2}\right),
\end{gathered}
$$


where $u(z)=0$ for $0<z<k$. We highlight that (38) is nearly identical to (20) from the one-dimensional case, except for the rows of stimulators above and below in an additional dimension. Because of this similarity, we can follow an analogous set of steps to those which start with (20) and end with the control strategy (31), i.e. writing and substituting the order $\epsilon$ asymptotic expansion of $\dot{\psi}_{r_{x}, r_{y}}$ into (38), taking the spatial average of the resulting equation, appropriately time shifting the terms of the integrand, and solving the calculus of variations problem to derive the following approximately optimal control application:

$$
\begin{aligned}
u^{*}(t) & =\frac{-\zeta\left(\mathrm{APD}_{n}-\mathrm{APD}_{n-1}\right) g(t)}{2 \int_{0}^{k} g^{2}(\tau) d \tau} \\
g(t) & =\frac{1}{L^{2}} \int_{-L / 2}^{L / 2} \int_{-L / 2}^{L / 2}\left(\sum_{j=-\beta_{y}^{-}}^{\beta_{y}^{+}} \sum_{i=-\beta_{x}^{-}}^{\beta_{x}^{+}} \mathcal{I}\left(i L-r_{x}, j L-r_{y}, t-r_{x} / c+i L / c\right)\right) d r_{x} d r_{y} .
\end{aligned}
$$

Intuitively, the control strategy (39) is similar in spirit to the one dimensional control control strategy (31) taking into account the net effect of all stimulators in the domain on a square patch of tissue. In this example, boundary conditions of (35) are not periodic, so $g(t)$ will not necessarily be the same for all locations in the domain. Recall that in Figure 9 the iIRC for a given location is approximately zero for locations about 0.8 $\mathrm{cm}$ away. As a result, any stimulator index for which $\left|i L-r_{x}\right|>0.8 \mathrm{~cm}$ or $\left|j L-r_{y}\right|>0.8 \mathrm{~cm}$ in the argument of the iIRC from (39) will have a negligable effect on the integrand. On the square domain, $\left|r_{x}\right| \leq L / 2$ and $\left|r_{y}\right| \leq L / 2$ so that any stimulator index greater than $0.8 / L+1 / 2$ in any direction will not greatly alter the integrand from (39). As a result, choosing a stimulator in the center of the domain for calculation of (39) will yield a stimulus $u^{*}(t)$ which will eliminate alternans at all locations sufficiently far from the boundaries. Panel A of Figure 9 shows the resulting optimal stimuli for different choices of $L$ and $\zeta=1.5$. The shape of each stimulation is approximately the same, with a magnitude that is approximately proportional to $L^{2}$. For simulations of (35), independent and identically distributed noise is added to the voltage variable. The

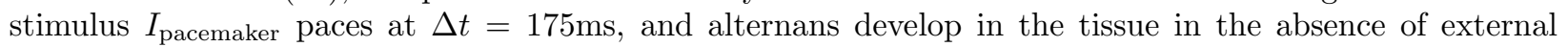
control as illustrated in panel $\mathrm{C}$ of Figure 11. The control strategy (39) is applied to the system with spacing $L=0.5 \mathrm{~cm}$ and panels $\mathrm{D}$ and E show the difference between successive APDs immediately after and $1050 \mathrm{~ms}$ after the control strategy is turned on, respectively. The first application of the control stimulus almost completely eliminates alternans in the system and soon after, APDs are nearly identical for each beat. Panels $\mathrm{F}$ and $\mathrm{G}$ show the transmembrane voltage and the applied control, respectively, at a location near the middle of the tissue. Similar to the one-dimensional case, the first control application is large compared to the succesive control applications which maintain the alternans-free state. We also test the non-optimal control strategy (32) [46] in the two-dimensional simulations. The average power to maintain an alternans-free state for each of these strategies is shown in panel B, where the energy usage is given by $E=\sum_{i} \sum_{j} \int u_{i, j}^{2}(t) d t$, and the average power is the energy usage divided by the total time. In these simulations, the optimal strategy is more than 300 times more efficient than the non-optimal strategy for each value of $L$. Furthermore, the non-optimal strategy does not work for a spacing of $L=1 \mathrm{~cm}$; the optimal strategy with $L=1 \mathrm{~cm}$ is significantly better than the non-optimal strategy with $L=0.1 \mathrm{~cm}$. This is a marked difference from the one-dimensional case, where the optimal strategy performed approximately 3 times better than the non-optimal strategy. We note that in [46] the authors found a comparable increase of the strategy (32) when moving from one to two dimensions. It is possible that in two dimensions, (32) is not very stable and is less efficient as a result. The optimal control strategy, which explicitly controls the isostable coordinate of the system, does not suffer from comparable losses in efficiency. 

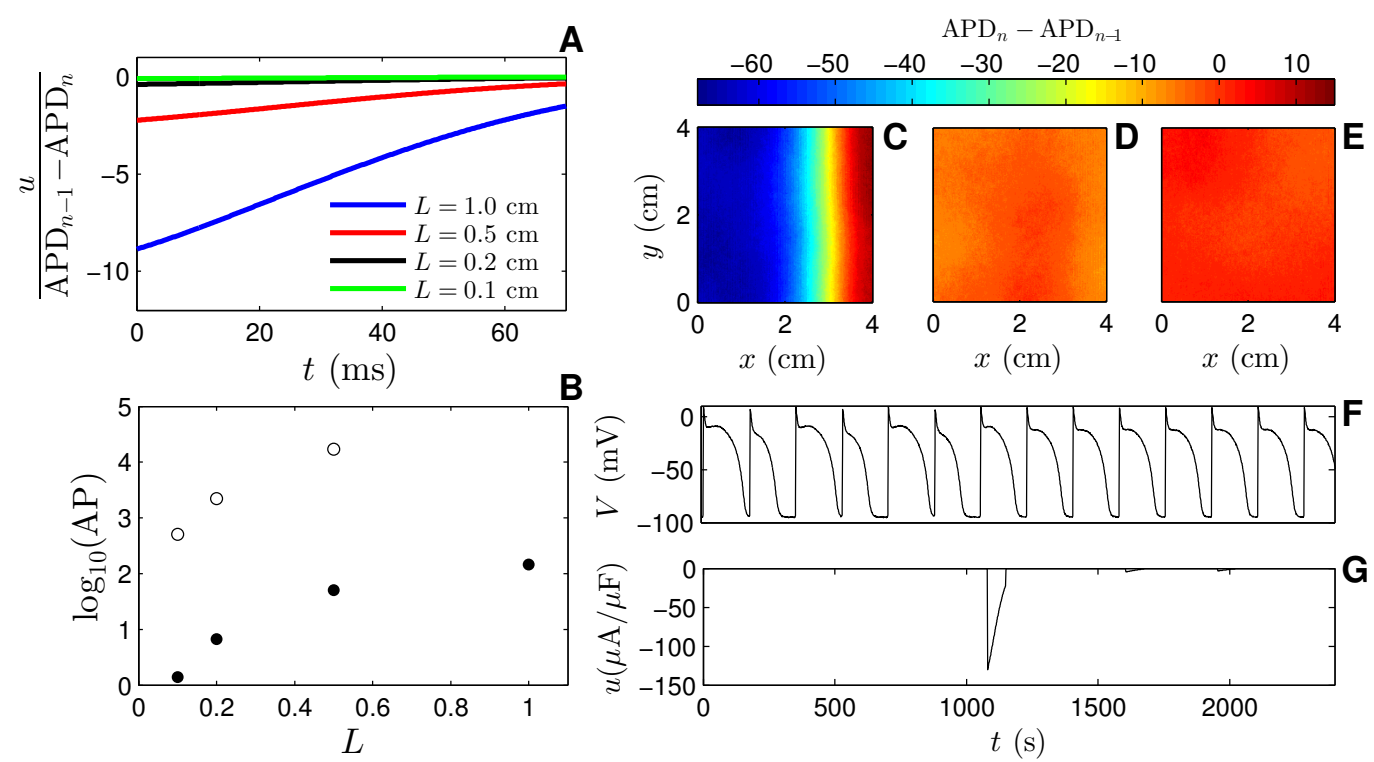

FIG. 11. Panel A shows the stimulus (39) calculated for different values of $L$. Panel B gives a comparison of the average power required to maintain an alternans-free state for the optimal strategy (39) (dots) and voltage difference strategy (32) (open circles). Panels C, D, and E show the difference between successive action potentials immediately prior to, immediately after, and $1050 \mathrm{~ms}$ after the optimal control strategy is turned on. Panels $\mathrm{F}$ and $\mathrm{G}$ show the transmembrane voltage and applied control at a location near the center of the tissue. The control is turned on at $t \approx 1000 \mathrm{~ms}$.

\section{CONCLUSIONS}

Cardiac alternans have been implicated as a precursor to cardiac fibrillation, and eliminating them could prevent fibrillation for those who are at risk for this life threatening arrhythmia. In this work, we have investigated strategies for elimination of alternans in a PDE model of cardiac tissue. The physiologically complicated models of alternans considered here are difficult to work with directly, but isostable reduction is shown to be useful starting point from which to develop nearly optimal control strategies for the stabilization of the underlying period-1 behavior and subsequent termination of alternans.

The control strategies developed in this work eliminate alternans using significantly less energy than a previously proposed method [46]. Furthermore, while [46] uses chaos control methods akin to delayed feedback control [42], [43], a model independent strategy which uses continuous state feedback to stabilize an unstable periodic orbit, the alternans control strategy developed in this work does not require continuous feedback. Rather, it only needs measurement action potential durations at each control node after an iIRC has been measured.

Isostable reduction can be a powerful strategy for both understanding and controlling systems with complicated and high dimensional dynamics. The isostable reduction strategy described in this work does not require the state dynamics to collapse to a one-dimensional manifold. For the calculation of the iIRCs in this work, the state variables differ on a beat-to-beat basis, but the resulting isostable response curves show little variability between beats. The isostable reduction methodology was illustrated in one- and two-dimensional models of cardiac tissue, but this strategy would be straightforward to extend to three dimensional models with more complicated spatial geometries at the expense of higher computational effort.

The ability to determine iIRCs is essential to the isostable reduction strategy and the subsequent control strategy for eliminating alternans, a calculation which we have demonstrated by using the adjoint equation (7) in a numerical model. However, in live tissue experiments it is likely that we would not have access the full dynamic equations required to use adjoint methods. We suggest a protocol akin to the direct method for calculating phase response curves [26], [37] by which an iIRC could be measured in vitro. Numerically, we find that iIRCs measured directly are similar to those calculated from (7), yielding control stimuli which terminate alternans effectively. In this manuscript, we assume the ability to inject current directly into the cellular 
membrane, something which would not be feasible in practice. Nevertheless, the method of calculating iIRCs only requires the knowledge of the system to a given type of perturbation, and the measurement strategies presented in Section V would extend naturally if, for instance, stimulation is applied with a bipolar electrode.

This control strategy is certainly not without limitations. Foremost, it would be difficult to implement control with a grid of actuators for in vivo aplications as in Section VI, and it would be of interest to adapt this control strategy to utilize the minimum number of control nodes possible to eliminate alternant behavior. Furthermore, the present control strategy does not explicitly take into account heterogeneities at the cellular level or more realistic spatial geometries in live cardiac tissue, both of which may alter the efficacy of the proposed control algorithm. Recent studies have implicated alternant calcium dynamics as a driving force behind APD alternans [20], [45], [10]. While the dynamics of the models considered in this work are deterministic, at a mircoscopic cellular level, calcium dynamics are often modeled as stochastic events [38], [47], [52]. While current isostable reduction techniques cannot explicitly handle stochastic dynamics, their continued development with this goal in mind would be of interest in the investigation of calcium alternans.

Support for this work by National Science Foundation Grant NSF-1363243 and NSF-1602841 is gratefully acknowledged.

\section{Appendix A: An Intuitive Example of Isostable Coordinates: Illustration For a Planar ODE}

While the definition of isostable coordinates is necessarily different between ODEs (as presented in [36]) and PDEs (as presented in the current manuscript and in [60]), much of the underlying intuition about isostables is the same for each type of system. Here we provide an illustrative example of isostable coordinates in a planar excitable ODE system similar to the analysis that originally appears in [58].

Consider the following ODE

$$
\dot{x}=F(x), \quad x \in \mathbb{R}^{2} .
$$

Suppose that (A1) has a stable fixed point at $x_{0}$. Then near the fixed point

$$
\dot{x}=A\left(x-x_{0}\right)+\mathcal{O}\left(\left(x-x_{0}\right)^{2}\right),
$$

where $A \in \mathbb{R}^{2 \times 2}$ is the Jacobian of the vector field evaluated at $x_{0}$. Suppose that $\lambda_{1}$ and $\lambda_{2}$ are the eigenvalues of $A$ corresponding to eigenvectors $v_{1}$ and $v_{2}$, and that $\left|\lambda_{1}\right|<\left|\lambda_{2}\right|$. Near the fixed point, higher order terms are negligible, and based on the solution of the linear equation (A2) one can show that in the limit as time approaches infinity,

$$
\left(\varphi(t, x(0))-x_{0}\right) \propto v_{1},
$$

where $\varphi(t, x(0))$ is a solution to (A1) with initial condition $x(0)$ (also known as the flow). Equation (A3) results from components in the $v_{2}$ direction decaying faster than component in the $v_{1}$ direction. We can use this information to define scalar isostable coordinates in the basin of attraction of $x_{0}$ (c.f. [36]. [58]):

$$
\psi(x)=\frac{1}{\lambda_{1}} \log \left(\lim _{t \rightarrow \infty}\left[e^{-\lambda_{1} t} z^{T}\left(\varphi(t, x)-x_{0}\right)\right]\right)
$$

Here, $z \in \mathbb{R}^{2}$ is some observable for which $z^{t} v_{1} \neq 0$. Note the similarity between the definition (A4) for ODEs and definition (4) for PDEs from the main text. Intuitively, inside the brackets of (A4), an exponentially growing term is multiplied by another term that is shrinking exponentially at the same rate; as time goes to infinity, this product approaches a nonzero constant. Taking the logarithm and normalizing by $\lambda_{1}$ provides a coordinate system for which $d \psi / d t=1$.

As an illustration, consider a FitzHugh-Nagumo based model for an excitable system, which can be used as a minimal model to represent the activity of a single cardiomyocyte [50]:

$$
\begin{aligned}
& \dot{V}=c_{1} V(V-a)(1-V)-c_{2} V w, \\
& \dot{w}=b(V-d w) .
\end{aligned}
$$

Here $V$ represents the transmembrane voltage, $w$ is a gating variable, $a=0.13, b=0.013, c_{1}=0.26, c_{2}=0.1$, and $d=1$. This system has a stable fixed point at $x_{0}=[0,0]^{T}$, and because it is excitable, a perturbation 
above a certain threshold from the fixed point can yield an excursion well beyond that threshold. Linearizing about this fixed point yields eigenvalues $\lambda_{1}=-0.013$ and $\lambda_{2}=-0.0338$. Using this information to calculate isostables for this system and taking $z^{T}=[11]$ yields the left panel of Figure A1. The right panel gives the trajectories of three initial conditions which start on the same isostable value.
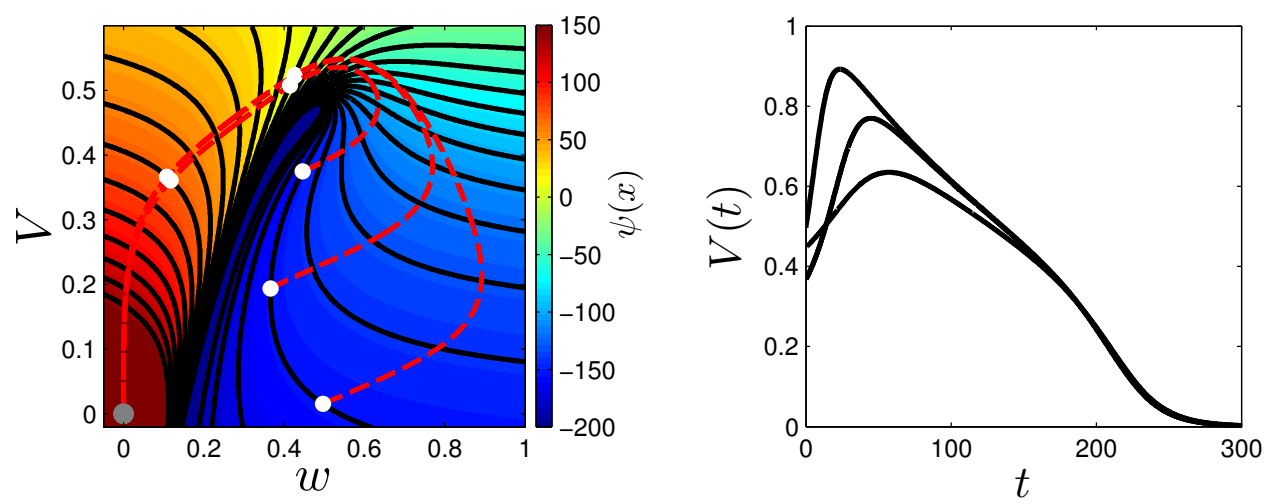

FIG. A1. The left panel shows isostable values defined by (A4) for the ODE model (A5). Black lines represent level sets and the fixed point is shown in gray. Three trajectories are plotted as dashed red lines, with white dots showing their locations for three different snapshots in time. The right panel shows the voltage trace for these same three trajectories. Notice that while the dynamics are different initially, they begin to converge on the way to the fixed point. These trajectories approach each other long before the trajectories reach the fixed point, even though $\left|\lambda_{1}\right|$ and $\left|\lambda_{2}\right|$ are similar in magnitude.

Viewing (A5) from the perspective of isostables, the trajectories which start on the same isostable begin to converge, even before the voltage-like variable reaches its resting value. This ODE example provides a basis for understanding the utility of isostable reduction in PDEs. By understanding the isostable field in of a complicated, infinite-dimensional PDE, we can work with it in a lower dimensional space as illustrated in Section II.

[1] C. C. Canavier. Phase-resetting as a tool of information transmission. Current Opinion in Neurobiology, 31:206$213,2015$.

[2] C. C. Canavier, F. G. Kazanci, and A. A. Prinz. Phase resetting curves allow for simple and accurate prediction of robust N:1 phase locking for strongly coupled neural oscillators. Biophysical Journal, 97(1):59-73, 2009.

[3] E. M. Cherry and F. H. Fenton. Visualization of spiral and scroll waves in simulated and experimental cardiac tissue. New Journal of Physics, 10(12):125016, 2008.

[4] E. M. Cherry, F. H. Fenton, and R. F. Gilmour Jr. Mechanisms of ventricular arrhythmias: a dynamical systems-based perspective. American Journal of Physiology-Heart and Circulatory Physiology, 302(12):H2451, 2012.

[5] D. J. Christini, M. L. Riccio, C. A. Culianu, J. J. Fox, A. Karma, and R. F. Gilmour Jr. Control of electrical alternans in canine cardiac Purkinje fibers. Physical Review Letters, 96(10):104101, 2006.

[6] R. H. Clayton, O. Bernus, E. M. Cherry, H. Dierckx, F. H. Fenton, L. Mirabella, A. V. Panfilov, F. B. Sachse, G. Seemann, and H. Zhang. Models of cardiac tissue electrophysiology: progress, challenges and open questions. Progress in Biophysics and Molecular Biology, 104(1):22-48, 2011.

[7] P. Constantin, C. Foias, B. Nicolaenko, and R. Temam. Integral Manifolds and Inertial Manifolds for Dissipative Partial Differential Equations, volume 70. Springer, New York, 1989.

[8] M. Courtemanche, L. Glass, and J. P. Keener. Instabilities of a propagating pulse in a ring of excitable media. Physical Review Letters, 70(14):2182, 1993.

[9] M. Courtemanche, J. P. Keener, and L. Glass. A delay equation representation of pulse circulation on a ring in excitable media. SIAM Journal on Applied Mathematics, 56(1):119-142, 1996.

[10] M. E. Díaz, S. C. O'Neill, and D. A. Eisner. Sarcoplasmic reticulum calcium content fluctuation is the key to cardiac alternans. Circulation Research, 94(5):650-656, 2004. 
[11] B. Echebarria and A. Karma. Instability and spatiotemporal dynamics of alternans in paced cardiac tissue. Physical Review Letters, 88(20):208101, 2002.

[12] G. B. Ermentrout, B. Beverlin II, T. Troyer, and T. I. Netoff. The variance of phase-resetting curves. Journal of Computational Neuroscience, 31(2):185-197, 2011.

[13] G. B. Ermentrout and N. Kopell. Multiple pulse interactions and averaging in systems of coupled neural oscillators. Journal of Mathematical Biology, 29(3):195-217, 1991.

[14] F. Fenton, E. Cherry, H. Hastings, and S. Evans. Multiple mechanisms of spiral wave breakup in a model of cardiac electrical activity. Chaos, 12:852-892, 2002.

[15] C. Foias, G. R. Sell, and R. Temam. Inertial manifolds for nonlinear evolutionary equations. Journal of Differential Equations, 73(2):309-353, 1988.

[16] J. J. Fox, J. L. McHarg, and R. F. Gilmour. Ionic mechanism of electrical alternans. American Journal of Physiology-Heart and Circulatory Physiology, 282(2):H516-H530, 2002.

[17] L. H. Frame and M. B. Simson. Oscillations of conduction, action potential duration, and refractoriness. a mechanism for spontaneous termination of reentrant tachycardias. Circulation, 78(5):1277-1287, 1988.

[18] A. Garzón, R. O. Grigoriev, and F. H. Fenton. Model-based control of cardiac alternans in Purkinje fibers. Physical Review E, 84(4):041927, 2011.

[19] A. Garzón, R. O. Grigoriev, and F. H. Fenton. Continuous-time control of alternans in long Purkinje fibers. Chaos: An Interdisciplinary Journal of Nonlinear Science, 24(3):033124, 2014.

[20] J. I. Goldhaber, L. H. Xie, T. Duong, C. Motter, K. Khuu, and J. N. Weiss. Action potential duration restitution and alternans in rabbit ventricular myocytes the key role of intracellular calcium cycling. Circulation Research, 96(4):459-466, 2005.

[21] R. A. Gray, A. M. Pertsov, and J. Jalife. Spatial and temporal organization during cardiac fibrillation. Nature, 392(6671):75-78, 1998.

[22] J. Guckenheimer and P. Holmes. Nonlinear Oscillations, Dynamical Systems, and Bifurcations of Vector Fields, volume 42. Springer Verlag, New York, 1983.

[23] K. Hall, D. J. Christini, M. Tremblay, J. J. Collins, L. Glass, and J. Billette. Dynamic control of cardiac alternans. Physical Review Letters, 78(23):4518-4521, 1997.

[24] F. C. Hoppensteadt and E. M. Izhikevich. Weakly Connected Neural Networks. Springer, New York, 1997.

[25] H. Ito and L. Glass. Theory of reentrant excitation in a ring of cardiac tissue. Physica D, 56(1):84-106, 1992.

[26] E. M. Izhikevich. Dynamical Systems in Neuroscience: The Geometry of Excitability and Bursting. MIT Press, London, 2007.

[27] U. B. Kanu, S. Iravanian, R. F. Gilmour, and E. J. Christini. Control of action potential duration alternans in canine cardiac ventricular tissue. Biomedical Engineering, IEEE Transactions on, 58(4):894-904, 2011.

[28] J. P. Keener. Principles of Applied Mathematics. Addison-Wesley, Redwood City, California, 1988.

[29] J. K. Kim, D. B. Forger, M. Marconi, D. Wood, A. Doran, T. Wager, C. Chang, and K. M. Walton. Modeling and validating chronic pharmacological manipulation of circadian rhythms. CPT: Pharmacometrics 85 Systems Pharmacology, 2(7):1-11, 2013.

[30] D. Kirk. Optimal Control Theory. Dover Publications, New York, 1998.

[31] Y. Kobayashi, W. Peters, S. S. Khan, W. J. Mandel, and H. S. Karagueuzian. Cellular mechanisms of differential action potential duration restitution in canine ventricular muscle cells during single versus double premature stimuli. Circulation, 86(3):955-967, 1992.

[32] T. Krogh-Madsen, A. Karma, M. L. Riccio, P. N. Jordan, D. J. Christini, and R. F. Gilmour Jr. Off-site control of repolarization alternans in cardiac fibers. Physical Review E, 81(1):011915, 2010.

[33] Y. Kuramoto. Chemical Oscillations, Waves, and Turbulence. Springer-Verlag, Berlin, 1984.

[34] M. Li and N. F. Otani. Controlling alternans in cardiac cells. Annals of Biomedical Engineering, 32(6):784-792, 2004.

[35] S. Luther, F. Fenton, B. Kornreich, A. Squires, P. Bittihn, D. Hornung, M. Zabel, J. Flanders, A. Gladuli, L. Campoy, E. Cherry, B. Luther, G. Hasenfuss, V. Krinsky, A. Pumir, R. Gilmour Jr, and E. Bodenschatz. Low-energy control of electrical turbulence in the heart. Nature, 475:235-241, 2011.

[36] A. Mauroy, I. Mezić, and J. Moehlis. Isostables, isochrons, and Koopman spectrum for the action-angle representation of stable fixed point dynamics. Physica D: Nonlinear Phenomena, 261:19-30, 2013.

[37] T. Netoff, M. A. Schwemmer, and T. J. Lewis. Experimentally estimating phase response curves of neurons: theoretical and practical issues. In Phase Response Curves in Neuroscience, pages 95-129. Springer, 2012.

[38] M. Nivala and Z. Qu. Calcium alternans in a couplon network model of ventricular myocytes: role of sarcoplasmic reticulum load. American Journal of Physiology-Heart and Circulatory Physiology, 303(3):H341-H352, 2012.

[39] J. B. Nolasco and R. W. Dahlen. A graphic method for the study of alternation in cardiac action potentials. Journal of Applied Physiology, 25(2):191-196, 1968.

[40] S. A. Oprisan. A geometric approach to phase resetting estimation based on mapping temporal to geometric phase. In Phase Response Curves in Neuroscience, pages 131-162. Springer, 2012.

[41] S. A. Oprisan, V. Thirumalai, and C. C. Canavier. Dynamics from a time series: can we extract the phase resetting curve from a time series? Biophysical Journal, 84(5):2919-2928, 2003. 
[42] K. Pyragas. Continuous control of chaos by self-controlling feedback. Physics letters A, 170(6):421-428, 1992.

[43] K. Pyragas and A. Tamaševičius. Experimental control of chaos by delayed self-controlling feedback. Physics Letters A, 180(1):99-102, 1993.

[44] Z. Qu, A. Garfinkel, P. Chen, and J. N. Weiss. Mechanisms of discordant alternans and induction of reentry in simulated cardiac tissue. Circulation, 102(14):1664-1670, 2000.

[45] Z Qu, M. Nivala, and J. N. Weiss. Calcium alternans in cardiac myocytes: order from disorder. Journal of Molecular and Cellular Cardiology, 58:100-109, 2013.

[46] W.J. Rappel, F. Fenton, and A. Karma. Spatiotemporal control of wave instabilities in cardiac tissue. Physical Review Letters, 83(2):456, 1999.

[47] J. G. Restrepo and A. Karma. Spatiotemporal intracellular calcium dynamics during cardiac alternans. Chaos: An Interdisciplinary Journal of Nonlinear Science, 19(3):037115, 2009.

[48] A. J. Roberts. Macroscale, slowly varying, models emerge from the microscale dynamics. IMA Journal of Applied Mathematics, 80:1492-1518, 2015.

[49] B. Rodriguez, L. Li, J. C. Eason, I. R. Efimov, and N. A. Trayanova. Differences between left and right ventricular chamber geometry affect cardiac vulnerability to electric shocks. Circulation Research, 97(2):168-175, 2005.

[50] J. Rogers and A. McCulloch. A collocation-Galerkin finite element model of cardiac action potential propagation. IEEE Transactions on Biomedical Engineering, 41:743-757, 1994.

[51] D. S. Rosenbaum, L. E. Jackson, J. M. Smith, H. Garan, J. N. Ruskin, and R. J. Cohen. Electrical alternans and vulnerability to ventricular arrhythmias. New England Journal of Medicine, 330(4):235-241, 1994.

[52] R. Rovetti, X. Cui, A. Garfinkel, J. N. Weiss, and Z. Qu. Spark-induced sparks as a mechanism of intracellular calcium alternans in cardiac myocytes. Circulation Research, 106(10):1582-1591, 2010.

[53] F. H. Sieling, S. Archila, R. Hooper, C. C. Canavier, and A. A. Prinz. Phase response theory extended to nonoscillatory network components. Physical Review E, 85(5):056208, 2012.

[54] N. Trayanova. Concepts of ventricular defibrillation. Philosophical Transactions of the Royal Society of London A: Mathematical, Physical and Engineering Sciences, 359(1783):1327-1337, 2001.

[55] M. A. Watanabe, F. H. Fenton, S. J. Evans, H. M. Hastings, and A. Karma. Mechanisms for discordant alternans. Journal of Cardiovascular Electrophysiology, 12(2):196-206, 2001.

[56] J. N. Weiss, A. Karma, Y. Shiferaw, P. Chen, A. Garfinkel, and Z. Qu. From pulsus to pulseless: the saga of cardiac alternans. Circulation Research, 98(10):1244-1253, 2006.

[57] D. Wilson, A. B. Holt, T. I. Netoff, and J. Moehlis. Optimal entrainment of heterogeneous noisy neurons. Frontiers in Neuroscience, 9, 2015.

[58] D. Wilson and J. Moehlis. An energy-optimal methodology for synchronization of excitable media. SIAM Journal on Applied Dynamical Systems, 13(2):944-957, 2014.

[59] D. Wilson and J. Moehlis. Extending phase reduction to excitable media: Theory and applications. SIAM Review, 57(2):201-222, 2015.

[60] D. Wilson and J. Moehlis. Isostable reduction with applications to time-dependent partial differential equations. Phys. Rev. E, 94:012211, 2016.

[61] A. Winfree. The Geometry of Biological Time. Springer Verlag, New York, second edition, 2001. 